\title{
SOME PROPERTIES OF THE FOURIER TRANSFORM ON SEMI-SIMPLE LIE GROUPS. PART II $\left({ }^{1}\right)$
}

\author{
BY

\section{EHRENPREIS AND F. I. MAUTNER}

This is a continuation of Part I (see Ehrenpreis and Mautner [1]). Throughout the present Part II, $G$ denotes again the group of all conformal mappings of the interior of the unit circle. In Part I we studied mainly functions $f(g)$ on $G$ which satisf $f\left(k g k^{\prime}\right)=f(g)$ for all $g \in G, k$ and $k^{\prime} \in K$, where $K$ is again the subgroup of rotations about the origin. In the present Part II we shall study the behavior of functions and function-spaces on $G$ under the Fourier transform on $G$ without that restriction. For this purpose it is useful to consider for each pair of fixed integers $m, n$ the class of complex valued functions $f(g)$ on $G$ which satisfy

$$
f\left(k_{\theta}^{-1} g k_{\vartheta}^{-1}\right)=e^{2 \pi i n \theta} \cdot f(g) \cdot e^{2 \pi i m \vartheta}
$$

for all elements $g$ of $G$ and all rotations $k_{\theta}, k_{\vartheta}$ through arbitrary angles $\theta$, $\vartheta \geqq 0,<1$.

Definition 0.1. The class of all complex-valued functions $f(g)$ on $G$ which satisfy $(0.1)$ for fixed integers $m, n$ will be called the class of spherical functions of type $m, n$.

Our method of studying a given class of functions on $G$ consists in first studying the subclass of all spherical functions of type $m, n$ and then summing (in some suitable sense) over $m$ and $n$. This approach is intrinsically connected with the structure of $G$ and its irreducible representations. Of course, Definition 0.1 depends on the particular choice of the maximal compact subgroup $K$. But since any two maximal compact subgroups of $G$ are conjugate in $G$, Definition 0.1 is invariant up to inner automorphisms of $G$.

We wish to study the structure of various group-algebras on $G$ such as the algebra $D$ (with convolution as product) of all indefinitely differentiable functions of compact support on $G$. We shall succeed in characterizing completely the Fourier transform of $D$, by proving an analogue for $G$ of the Paley-WienerSchwartz theorem. Thus $D$ will be shown to be naturally isomorphic (via the Fourier-transform) to a completely determined matrix-algebra where the matrix-coefficients are entire functions of exponential type satisfying simple

Received by the editors October 14, 1955.

(1) This work was supported by Grant NSF5-G1010 from the National Science Foundation and Contract DA-36-034-ORD-1622RD with the Office of Ordnance Research through the Institute for Advanced Study. Both authors are on leave of absence from the Johns Hopkins University. The second named author is a fellow of the John Simon Guggenheim Memorial Foundation. 
functional equations. Thus we have a complete characterization of a groupalgebra of a noncompact semi-simple Lie group.

To obtain this characterization of $D$ we study first the subspace $D_{m n}$ of $D$ consisting of all spherical functions of type $m, n$ contained in $D$. It will be seen in $\$ 2$ that for fixed $m, n$, the Fourier transform of $D_{m n}$ consists of all numerical entire functions of exponential type satisfying certain simple conditions. We thus obtain a very well known class of analytic functions, which can be characterized alternatively as an explicitly defined subspace of the Fourier transform on the real line of the class $D$ of all indefinitely differentiable functions of compact support on the additive group of real numbers. Thus we shall obtain in $\S 2$ a very complete description of $D_{m n}$ and its Fourier transform, and also relate it to the space $D$ of Schwartz on the real line.

Using the results of $\S 2$ about $D_{m n}$ we shall characterize rather completely in $\$ 3$ the whole algebra $D$ and its Fourier transform. This will enable us to study the dual space $D^{\prime}$ of $D$, the space of distributions in the sense of L. Schwartz [4] on the real analytic manifold $G$ (see $\$ 4$ below). We shall also introduce the space $E$ of all indefinitely differentiable functions on $G$ (with no restriction on their supports) as a certain topological vector-space. The dual space $E^{\prime}$ of $E$ is the space of all distributions of compact supports on $G$. Since the convolution of any two distributions of compact supports exists, is continuous and is again $\in E^{\prime}$, hence $E^{\prime}$ is a topological algebra. It appears to be a particularly useful and interesting group-algebra. On the one hand it contains all integrable functions of compact supports, but also the associative enveloping algebra of the Lie algebra of $G$, whose great importance has been brought out in the work of Harish-Chandra. On the other hand we shall give in $\$ 4$ a complete characterization of $E^{\prime}$ as an explicit matrix-algebra where the matrix-coefficients are again entire functions of exponential type.

In $\S 5$ we study the subspaces $E_{m n}, E_{m n}^{\prime}$ of $E, E^{\prime}$ respectively, consisting of the spherical elements of type $m, n\left(\in E, E^{\prime}\right)$ in more detail. This leads to an analogue of L. Schwartz's [3] theory of mean periodic functions, in particular to an anlogue for spherical functions of type $m, n$, of L. Schwartz's main expansion theorem for mean periodic functions.

In $\S 6$ we introduce the notion of two-sided mean-periodicity, which is not restricted to spherical functions. We obtain an expansion theorem for twosided mean periodic functions by reducing the problem to spherical functions and applying the results of $\S 5$. It seems remarkable that there thus exists a three-dimensional (simple) Lie group for which an expansion theorem for mean periodic functions holds, while the corresponding problem for the additive group of Euclidean vector space is still unsolved for dimension greater than one.

Some remarks about notation. E denotes the space of all indefinitely differentiable functions on $G$ (this differs from the notation of Part I: the space of all indefinitely differentiable spherical functions of type 0,0 is now denoted 
by $E_{00}$, not by $E$ ). $D$ denotes the space of all indefinitely differentiable functions of compact support on $G$ (this differs again from the notation of Part I). By $E^{\prime}, D^{\prime}$ we denote their dual spaces.

$\varepsilon$ denotes the space of all indefinitely differentiable functions on the real line, $D$ the subspace of $\mathcal{E}$ of functions of compact support; $\mathcal{E}^{\prime}$ and $D^{\prime}$ are their dual spaces, so that $D^{\prime}$ is the space of all distributions on the real line in the sense of L. Schwartz, $\mathcal{E}^{\prime}$ the space of all distributions of compact support on the real line. For any $y(x) \in D$ we put

$$
Y(s)=\int_{-\infty}^{\infty} y(x) \exp \left[\left(s-\frac{1}{2}\right) x\right] d x
$$

so that $Y(s)$ is an entire function of exponential type rapidly decreasing on the line $R s=1 / 2$. We denote by $\widehat{D}$ the space of all $Y(s)$ so obtained (by the theorem of Paley-Wiener-Schwartz $\widehat{D}$ is the space of all entire functions of exponential type rapidly decreasing on $R s=1 / 2$ ). Similarly $\widehat{\mathcal{E}}^{\prime}$ is defined, so that

$$
\widehat{D}=\text { image of } \mathscr{D} \text { under }(0.2) \text { and } \widehat{\mathcal{E}}^{\prime}=\text { image of } \mathcal{E}^{\prime} \text { under }(0.2) \text {. }
$$

1. Preliminaries. Let $g$ be any element of $G$, then $g$ is determined by a pair of matrices

$$
\pm\left\|\begin{array}{ll}
\alpha & \beta \\
\bar{\beta} & \bar{\alpha}
\end{array}\right\|
$$

of determinant 1 and $g$ can be written as a product

$$
g=k g_{\zeta} k^{\prime} \text { with } g_{\zeta}=\left\|\begin{array}{cc}
\cosh \zeta & \sinh \zeta \\
\sinh \zeta & \cosh \zeta
\end{array}\right\| ; \quad k, k^{\prime} \in K .
$$

This has been used throughout Part I and will be used again throughout the present Part II.

For any $g \in G$ and any complex number $s$ we introduce as in $\S 1$ of Part I again the operator $U(g, s)$ acting on functions $a(\theta)$ of the angle $\theta$ by putting

$$
[U(g, s) a](\theta)=\left[\frac{d g \theta}{d \theta}\right]^{s} a(g \theta) .
$$

We shall use again the inner products

$$
u_{m n}(g, s)=\left\langle U(g, s) e^{2 \pi i m \theta}, e^{2 \pi i n \theta}\right\rangle=\int_{0}^{1}\left[\frac{d g \theta}{d \theta}\right]^{8} e^{2 \pi i m g \theta} e^{-2 \pi i n \theta} d \theta .
$$

Lemma 1.1. For fixed $m, n$ and $s$ the function $u_{m n}(g, s)$ of $g$ is spherical of type $-m,-n$ in the sense of Definition 0.1 above. For fixed $m, n$ it is real analytic simultaneously in $g$ and $s$. For fixed $m, n$ and $g, u_{m n}\left(g_{5}, s\right)$ is an entire function of $s$ of exponential type $\leqq 2 \zeta$.

Proof. Let $k \vartheta$ be again the rotation through the angle $\vartheta$. Then 


$$
u_{m n}\left(k_{\vartheta} g, s\right)=\left\langle U\left(k_{\vartheta} g, s\right) e^{2 \pi i m \theta}, e^{2 \pi i n \theta}\right\rangle=\left\langle U(g, s) e^{2 \pi i m \theta}, U(k \vartheta, s)^{*} e^{2 \pi i n \theta}\right\rangle .
$$

But $U(k, s)$ is a unitary operator on $L_{2}(0 \leqq \theta<1 ; d \theta)$ for every $k \in K$ and every complex number $s$. Hence we obtain

$$
\left\langle U(g, s) e^{2 \pi i m \theta}, U\left(k_{-\vartheta}, s\right) e^{2 \pi i n \theta}\right\rangle=\left\langle U(g, s) e^{2 \pi i m \theta}, e^{2 \pi i n(\theta-\vartheta)}\right\rangle=e^{2 \pi i n \vartheta} u_{m n}\left(g_{2} s\right) .
$$

Similarly one verifies $u_{m n}\left(g k_{\vartheta}, s\right)=e^{2 \pi i m^{\vartheta}} u_{m n}(g, s)$.

It is clear from (1.2) that $u_{m n}(g, s)$ is for fixed $m$ and $n$ a real analytic function on the Cartesian product of $G$ with the $s$-plane, and is an entire function of $s$ for fixed $m, n$, and $g$. To prove that $u_{m n}\left(g_{5}, s\right)$ is an entire function of $s$ of exponential type $\leqq 2 \zeta$, note that (1.2) implies that $\left|u_{m n}(g, s)\right| \leqq u_{00}(g, \sigma)$ $=\phi(g, \sigma)$. By Theorem 1 of Part I, $\phi\left(g_{5}, s\right)$ is an entire function of $s$ of exponential type $\leqq 2 \zeta$, hence so is $u_{m n}\left(g_{\zeta}, s\right)$.

Remark. We shall use the following definition of exponential type throughout, which we have already used in Part I: An entire function $F(s)$ is of exponential type $\leqq r$, if $F(s)=O(\exp (r+\epsilon)|s|)$ for every $\epsilon>0$. The smallest value of $r$ for which this holds is the exponential type of $F(s)$. It can be shown that for functions $F(s)$ which satisfy $F(1 / 2+i t)=O(1)$ we even have $F(s)=O(\exp (r|s|))$ for this smallest value of $r$.

LEMMA 1.2. Let g vary over a given compact subset of $G$ and $s$ over a given lefthalf-plane. Then for all integers $m, n$ and every positive integer $q$

$$
\text { and } \begin{aligned}
\left|u_{m n}(g, s)\right| & \leqq c_{q}\left(\frac{|m|+|s|+1}{1+|n|}\right)^{q} \\
\left|u_{n m}(g, s)\right| & \leqq c_{q}\left(\frac{m+|s|+1}{1+|n|}\right)^{q}
\end{aligned}
$$

where $c_{q}$ is a constant depending only on $q$ and the given compact set.

Proof. It follows from (1.2) that

$$
\begin{aligned}
2 \pi i n u_{m n}(g, s) & =-\int_{0}^{1}\left[\frac{d g \theta}{d \theta}\right]^{s} e^{2 \pi i m g \theta} \frac{d}{d \theta}\left[e^{-2 \pi i n \theta}\right] d \theta \\
& =\int_{0}^{1} \frac{d}{d \theta}\left\{\left[\frac{d g \theta}{d \theta}\right]^{s} e^{2 \pi i m \theta}\right\} e^{-2 \pi i n \theta} d \theta \\
& =\int_{0}^{1}\left\{2 \pi i m\left[\frac{d g \theta}{d \theta}\right]^{s+1}+s\left[\frac{d g \theta}{d \theta}\right]^{s-1} \frac{d^{2} g \theta}{d \theta^{2}}\right\} e^{2 \pi i m \theta \theta} e^{-2 \pi i n \theta} d \theta .
\end{aligned}
$$

Now $d g \theta / d \theta$ and $d^{2} g \theta / d \theta^{2}$ are bounded as $g$ remains in a compact set; hence $[d g \theta / d \theta]^{s \pm 1}$ is bounded if in addition $R s$ is bounded from above. Hence

$$
\left|2 \pi m u_{m n}(g, s)\right| \leqq \int_{0}^{1}\left\{|m| c^{\prime}+|s| c^{\prime \prime}\right\} d \theta \leqq c^{\prime \prime \prime}(|m|+|s|) .
$$


Repeating, we obtain

$$
\begin{aligned}
(2 \pi i n)^{2} u_{m n}(g, s) & =-\int_{0}^{1}\left\{2 \pi i m\left[\frac{d g \theta}{d \theta}\right]^{s+1}+s\left[\frac{d g \theta}{d \theta}\right]^{s-1} \frac{d^{2} g \theta}{d \theta^{2}}\right\} e^{2 \pi i m g \theta} \frac{d}{d \theta}\left(e^{-2 \pi i n \theta}\right) d \theta \\
= & \int_{0}^{1}\left\{2 \pi i m\left[\frac{d g \theta}{d \theta}\right]^{s+2}+s\left[\frac{d g \theta}{d \theta}\right]^{s} \frac{d^{2} g \theta}{d \theta^{2}}\right\} 2 \pi i m e^{2 \pi i m g \theta} e^{-2 \pi i n \theta} d \theta \\
& +\int_{0}^{1}\left\{2 \pi i m(s+2)\left[\frac{d g \theta}{d \theta}\right]^{s+1} \frac{d^{2} g \theta}{d \theta^{2}}+s(s-1)\left[\frac{d g \theta}{d \theta}\right]^{s-2}\left(\frac{d^{2} g \theta}{d \theta}\right)^{2}\right. \\
& \left.+s\left[\frac{d g \theta}{d \theta}\right]^{s-1} \frac{d^{3} g \theta}{d \theta^{3}}\right\} e^{2 \pi i m g \theta} e^{-2 \pi i n \theta} d \theta .
\end{aligned}
$$

Therefore, as $g$ and $s$ vary as above,

$$
\begin{aligned}
(2 \pi n)^{2} u_{m n}(g, s) & \leqq(2 \pi|m|+|s|) 2 \pi|m|+2 \pi|m| \cdot|s+2|+\left|s^{2}-s+s\right| \\
& =4 \pi|m|^{2}+4 \pi|m s|+|s|^{2}+4 \pi m \leqq c_{2}(|m|+|s|+1)^{2} .
\end{aligned}
$$

This proves the part of the lemma for $u_{m n}(g, s)$ for $q=1,2$. The general $q$ can be treated similarly, as can $u_{n m}(g, s)$.

We shall also need to use the discrete series of irreducible unitary representations discovered by Bargmann [1, pp. 620-623]. For each positive integer $l$ let $H_{l}$ be the Hilbert space of those functions $a(z), b(z), \cdots$ defined and regular in the unit disc $|z|<1$ for which

(1.4) $\langle a, a\rangle_{l}=\left\{\begin{array}{l}\frac{l-1}{\pi} \iint_{|z|<1}\left(1-|z|^{2}\right)^{l-2}|a(z)|^{2} r d r d \theta \text { for } l>1, \\ \lim _{\epsilon \downarrow 0} \frac{l-1+\epsilon}{\pi} \iint_{|z|<1}\left(1-|z|^{2}\right)^{l-2+\epsilon}|a(z)|^{2} r d r d \theta \text { for } l=1\end{array}\right.$ is finite. As was shown by Bargmann [1, p. 621]

$$
\left\langle z^{m}, z^{m}\right\rangle_{l}=\left(\begin{array}{c}
l-1+m \\
m
\end{array}\right)^{-1}
$$

hence the functions

$$
\gamma_{n}(l) z^{n}\left(n=0,1,2, \cdots, \gamma_{n}(l)=\left(\begin{array}{c}
l-1+n \\
n
\end{array}\right)^{1 / 2}\right)
$$

form a complete orthonormal set in $H_{l}$. Following Bargmann we define for each $g \in G$ an operator $V^{+}(g, l)$ on $H_{l}$ by

$$
\left[V^{+}(g, l) a\right](z)=(d g z / d z)^{l} a(g z)
$$

and put 


$$
v_{m+l, n+l}^{+}(g, l)=\left\langle V^{+}(g, l) \gamma_{m}(l) z^{m}, \gamma_{n}(l) z^{n}\right\rangle_{l} .
$$

Then

$$
V^{+}(g, l) \gamma_{m}(l) z^{m}=\left(\frac{d g z}{d z}\right)^{l} \gamma_{m}(l)(g z)^{m}=\sum_{n=0}^{\infty} v_{n+l, m+l}^{+}(g, l)\left(\gamma_{n}(l) z^{n}\right)
$$

converges in the norm (1.4). Using Bargmann's [1] inequality (9.15) which asserts $|a(z)| \leqq\left(1-|z|^{2}\right)^{-l / 2}\|a\|_{l}$, we obtain pointwise convergence for each $z$ with $|z|<1$. Let us put at first formally $z=e^{2 \pi i \theta}$. Then we obtain the infinite series

$$
\sum_{n=0}^{\infty} v_{n+l, m+l}^{+}(g, l) \gamma_{n}(l) e^{2 \pi i n \theta}=e^{-2 \pi i l \theta} \gamma_{m}(l) \sum_{n=0}^{\infty} v_{n+l, m+l}^{+}(g, l) \frac{\gamma_{n}(l)}{\gamma_{m}(l)} e^{2 \pi i(n+l) \theta} .
$$

On the other hand for $z=e^{2 \pi i \theta}$

$$
\left(\frac{d g z}{d z}\right)^{l} \gamma_{m}(l)(g z)^{m}=\frac{e^{2 \pi i l o \theta}}{e^{2 \pi i l \theta}}\left(\frac{d g \theta}{d \theta}\right)^{l} e^{2 \pi i m \theta \theta} .
$$

But from Definition (1.2) it follows that

$$
\left(\frac{d g \theta}{d \theta}\right)^{l} e^{2 \pi i(m+l) \theta \theta}=\sum_{n=0}^{\infty} u_{n+l, m+l}(g, l) e^{2 \pi i(n+l) \theta}
$$

in the sense of convergence in the mean in $L_{2}(0 \leqq \theta \leqq 1 ; d \theta)$. Using Lemma 1.2 above, it follows that the last series converges even uniformly in $\theta$. Hence by comparison we may conclude that it is justified to put $z=e^{2 \pi i \theta}$ in the above infinite series for $V^{+}(g, l) \gamma_{m}(l) z^{m}$ and we obtain

$$
v_{n+l, m+l}^{+}(g, l)=\frac{\gamma_{m}(l)}{\gamma_{n}(l)} u_{n+l, m+l}(g, l), \quad m, n=0,1,2, \cdots ; l=1,2, \cdots,
$$

where

$$
\frac{\gamma_{m}(l)}{\gamma_{n}(l)}=\left(\begin{array}{c}
l-1+m \\
m
\end{array}\right)^{1 / 2}\left(\begin{array}{c}
l-1+n \\
n
\end{array}\right)^{-1 / 2}=\left(\frac{(l-1+m) ! n !}{(l-1+n) ! m !}\right)^{1 / 2} .
$$

Similarly one defines $V^{-}(g, l)$ as in $\S 9 \mathrm{~d}$ of Bargmann [1]. We put for $m, n$ $=0,1,2, \cdots$

$$
{v_{m+l, n+l}^{-}}^{-}(g, l)=\left\langle V^{-}(g, l) \gamma_{m}(l) z^{m}, \gamma_{n}(l) z^{n}\right\rangle
$$

and prove as above that

$$
\begin{aligned}
\bar{v}_{n+l, m+l}^{-}(g, l)= & \frac{\gamma_{m}(l)}{\gamma_{n}(l)} u_{-n-l,-m-l}(g, l) \\
& \text { for } m, n=0,1,2,3, \cdots ; l=1,2, \cdots
\end{aligned}
$$


For $l \geqq 1$ we shall write

$$
\omega_{m n}(l)= \begin{cases}\gamma_{m-l}(l) / \gamma_{n-l}(l) & \text { for } m \geqq l, n \geqq l, \\ \gamma_{-m-l}(l) / \gamma_{-n-l}(l) & \text { for }-m \geqq l ;-n \geqq l, \\ 0 & \text { otherwise }\end{cases}
$$

We shall now prove the analog of Lemma 1.2 for the $v_{m n}^{ \pm}(g, l)$ :

LEMma 1.3. Let $g$ vary over a given compact subset of $G$. Then for any positive integer $q$ we can find a positive number $c_{q}$ depending only on $q$ and the given compact set so that for $l=1,2,3, \cdots ; m, n=0,1,2,3, \cdots$, and $g$ in the given compact set, we have

$$
\text { and } \begin{aligned}
& \left|v_{m n}^{ \pm}(g, l)\right| \leqq c_{q}\left[\frac{(1+m+l)^{2}}{1+|n-l|}\right]^{q} \\
& \left|v_{n m}^{ \pm}(g, l)\right| \leqq c_{q}\left[\frac{(1+m+l)^{2}}{1+|n-l|}\right]^{q} .
\end{aligned}
$$

REMARK. Lemma 1.3 is not an immediate consequence of Lemma 1.2 because the $w_{m n}(l)$ grow too fast. Lemma 1.3 depends on the existence of the discrete series, and shows us that the $u_{m n}(g, s)$ ard actually very small at the integers.

Proof. We use the above expressions of the $v_{m n}^{ \pm}(g, l)$ as inner products. For simplicity we consider only $v_{m n}^{+}(g, l)$ since $v_{m n}^{-}(g, l)$ and $v_{n m}^{ \pm}(g, l)$ are handled similarly.

By (1.5) and (1.6) we have

$$
v_{m n}^{+}(g, l)=\gamma_{m-l}(l) \gamma_{n-l}(l) \iint\left(\frac{d g z}{d z}\right)^{l}(g z)^{m-l} \bar{z}^{n-l}\left(1-r^{2}\right)^{l-2} r d r d \theta
$$

where the integration is over the interior of the unit circle. Thus, proceeding as in the proof of Lemma 1.2, we have

$$
\begin{aligned}
& 2 \pi i(n-l) v_{m n}^{+}(g, l) \\
& =-\gamma_{m-l}(l) \gamma_{n-l}(l) \frac{l-1}{\pi} \iint\left(\frac{d g z}{d z}\right)^{l}(g z)^{m-l}\left(\overline{\left(\frac{\partial}{\partial \theta} z^{n-l}\right)}\right)\left(1-r^{2}\right)^{l-2} r d r d \theta \\
& =\gamma_{m-l}(l) \gamma_{n-l}(l) \frac{l-1}{\pi} \iint \bar{z}^{n-l} \frac{\partial}{\partial \theta}\left[\left(\frac{d g z}{d z}\right)^{l}(g z)^{m-l}\right]\left(1-r^{2}\right)^{l-2} r d r d \theta \\
& =\gamma_{m-l}(l) \gamma_{n-l}(l) \frac{l-1}{\pi} \iint \bar{z}^{n-l}\left\{l\left(\frac{d g z}{d z}\right)^{l-1} \frac{\partial}{\partial \theta}\left(\frac{d g z}{d z}\right)(g z)^{m-l}\right. \\
& \left.+\left(\frac{d g z}{d z}\right)^{l}(m-l)(g z)^{m-l-1} \frac{\partial}{\partial \theta}(g z)\right\}\left(1-r^{2}\right)^{l-2} r d r d \theta .
\end{aligned}
$$


By the Schwarz inequality,

$$
\begin{aligned}
& \left.\left|\gamma_{m-l}(l) \gamma_{n-l}(l) \frac{l-1}{\pi} \iint\right| z\right|^{n-l}\left|\frac{d g z}{d z}\right|^{l}|g z|^{m-l}\left(1-r^{2}\right)^{l-2} r d r d \theta \mid \\
& \leqq\left[\frac{l-1}{\pi} \iint \gamma_{n-l}^{2}(l) r^{2(n-l)}\left(1-r^{2}\right)^{l-2} r d r d \theta\right]^{1 / 2} \\
& \quad \times\left[\frac{l-1}{\pi} \iint \gamma_{m-l}^{2}(l)\left|\frac{d g z}{d z}\right|^{2 l}|g z|^{2(m-l)}\left(1-r^{2}\right)^{l-2} r d r d \theta\right]^{1 / 2}
\end{aligned}
$$

The first term on the right side of $(1.14)$ is 1 by the definition of $\gamma_{n-l}(l)$. To evaluate the second term, call $d \mu_{l}(z)=\left(1-r^{2}\right)^{l-2} r d r d \theta$. Then (see Bargmann [1]) $d \mu_{l}(z)=|d g z / d z|^{-2 l} d \mu_{l}(g z)$. Thus, the second term on the right side of equation (1.14) is just

$$
\left[\frac{l-1}{\pi} \iint \gamma_{m-l}^{2}(l) r^{2(n-l)}\left(1-r^{2}\right)^{l-2} r d r d \theta\right]^{1 / 2}=1 .
$$

By similar reasoning we obtain

$$
\left.\left|\frac{l-1}{\pi} \iint \gamma_{m-l}(l) \gamma_{n-l}(l)\right| \frac{d g z}{d z}\right|^{l}|g z|^{m-l-1}|z|^{n-l}\left(1-r^{2}\right)^{l-2} r d r d \theta \mid \leqq \frac{\gamma_{m-l}(l)}{\gamma_{m-l-1}(l)} .
$$

Now, it is clear from the definitions that

$$
\frac{\gamma_{m-l}(l)}{\gamma_{m-l-1}(l)}=\left(\frac{(m-1) !(m-l-1) !}{(m-2) !(m-l) !}\right)^{1 / 2} \leqq(m-1)^{1 / 2} \leqq m .
$$

Using these estimates in (1.13) and the facts that $|d g z / d z|,|d g z / d z|^{-1}$, $|\partial(d g z / d z) / \partial \theta|,|\partial(g z) / \partial \theta|$ are uniformly bounded for $|z| \leqq 1$ and $g$ in the fixed compact set, we obtain the result for $q=1$. The result for $q>1$ can be proven similarly, since several integrations by parts on the right side of (1.13) produce terms which can be handled like those above.

Leмma 1.4. Let $p$ be a non-negative integer. Then $u_{m n}(g, p)=0$ for all $g \in G$ if $m \geqq p$ and $n<0$ or if $m \leqq-p$ and $n>0$.

If $r$ is an integer $\leqq 1$ then $u_{m n}(g, r)=0$ for all $g \in G$ when $n \geqq 1-r$ and $m<0$ or when $n \leqq r-1$ and $m>0$. have

Proof. By definition of $U(g, s)$ and $u_{m n}(g, s)$ (see (1.1) and (1.2) above) we

$$
\left[\frac{d g \theta}{d \theta}\right]^{p} e^{2 \pi i m \theta \theta}=\sum_{n} u_{m n}(g, p) e^{2 \pi i n \theta} .
$$

Writing again $z=e^{2 \pi i \theta}$ we have as above 


$$
z^{p}\left[\frac{d g z}{d z}\right]^{p}(g z)^{m-p}=\sum_{n} u_{m n}(g, p) z^{n} .
$$

Now if $m \geqq p$ and $|z| \leqq 1$ the left side of this equation is regular in $z$, the right side is by Lemma 1.2 uniformly convergent in $|z| \leqq 1$, hence by the uniqueness of power series $u_{m n}(g, p)=0$ for all $g \in G$ when $n<0$ and $m \geqq p$. Now we use $\bar{u}_{m n}(g, \bar{s})=u_{-m,-n}(g, s)$ as follows immediately from (1.2) above and conclude that $u_{m n}(g, p)=0$ also for $m \leqq-p$ and $n>0$.

To prove the lemma for $r \leqq 1$, we use $\bar{u}_{m n}\left(g^{-1}, \bar{s}\right)=u_{n m}(g, 1-s)$ which is also an immediate consequence of (1.2) above.

We shall now make some remarks on the functional equation of $u_{m n}(g, s)$ : By equations (3.4), (3.5) and (3.6) of Part I, $u_{m n}(g, 1-s)=\chi_{m n}(s) u_{m n}(g, s)$, where $\left({ }^{2}\right)$ for $m \geqq 0$ and $n \geqq 0$,

$$
\chi_{m n}(s)=\prod_{j=1}^{n} \frac{j-s}{j-1+s} \prod_{j=1}^{m} \frac{j-1-s}{j-s}
$$

while

$$
\chi_{-m, n}(s)=(-1)^{m} \chi_{n m n}(s) \quad \text { and } \quad \chi_{m,-n}(s)=(-1)^{n} \chi_{m n}(s)
$$

define $\chi_{m n}(s)$ for all values of $m$ or $n$.

Assume first that $|n| \geqq|m|$. Then

$$
\chi_{m n}(s)= \pm \prod_{|m|+1}^{|n|}(j-s) \prod_{|m|+1}^{|n|} \frac{1}{j-1+s} .
$$

Thus, $\chi_{m n}(l+1)=0$ if $l$ is an integer, and $|n|>l,|m| \leqq l$. Since $u_{m n}(g, s)$ is an entire function of $s$, this means that $u_{m n}(g,-l)=0$ whenever $|m| \leqq l<|n|$. If $|m| \geqq|n|$, then

$$
\chi_{m n}(s)= \pm \prod_{|n|+1}^{|m|}(j-1+s) \prod_{|n|+1}^{|m|} \frac{1}{j-s} .
$$

We find that $\chi_{m n}(-l)=0$ for those integers $l$ such that $|n| \leqq l<|m|$. Thus, we must have $u_{m n}(l+1)=0$ whenever $|n| \leqq l<|m|$.

We shall also use some of the standard properties of vector- and operatorvalued integration. For details see Bourbaki [2].

Let $\mathfrak{T}$ be a complete, locally convex, topological vector space, $\Xi$ a locally compact Hausdorff space, $\mu$ a Radon measure on $\boldsymbol{\Xi}$, and $\mathrm{T}$ a map of $\boldsymbol{\Xi}$ into $\mathfrak{H}$. For simplicity, we suppose that $\mu$ is a positive measure, the general case being handled by taking linear combinations. By $L_{\infty}(\Xi)$ we denote the space of all complex valued bounded measurable functions on $\boldsymbol{\Xi}$.

We say that $\mathbf{T}$ is integrable (with respect to $\mu$ ) if

1. For any $\Lambda \in H^{\prime}$ (the dual of $\left.H\right), b \in L_{\infty}(\Xi) \int[\Lambda \cdot b(x) \mathrm{T}(x)] d \mu(x)$ exists in the sense of Lebesgue.

(2) The product $\prod_{j-1}^{0}$ is defined to be equal to one throughout. 
Denote by $J_{\mathrm{T}}(b)$ the map $\Lambda \rightarrow \int[\Lambda \cdot b(x) \mathrm{T}(x)] d \mu(x)$ of $\mathfrak{F C}^{\prime}$ to the complex numbers.

2. For each $b \in L_{\infty}, J_{\mathbf{T}}(b)$ defines a continuous linear mapping of $\mathcal{H}^{\prime}$ into the complex numbers, where $\mathfrak{H}^{\prime}$ is given the topology of uniform convergence on the compact sets of $\mathfrak{H C}$.

By 2. and well-known properties of topological vector spaces, we can find an $a \in H$ such that $J_{\mathrm{T}}(b)(\Lambda)=\Lambda \cdot a$ for all $\Lambda \in \mathcal{F}^{\prime}$. If we identify this $a$ with $J_{\mathrm{T}}(b)$, we see that $b \rightarrow J_{\mathrm{T}}(b)$ is a map of $L_{\infty}(\Xi)$ into $H$.

3. For any neighborhood $N$ of zero in $\mathcal{H}$ there is a compact set $C$ in $\Xi$ and an $\epsilon>0$ such that the conditions

$$
b \in L_{\infty}(\Xi) \sup _{x \in C}|b(x)| \leqq \epsilon, \quad \sup _{x \in \Xi}|b(x)| \leqq 1
$$

imply $J_{\mathrm{T}}(b) \in N$.

If $\mathrm{T}$ is integrable, we set $\int \mathrm{T} d \mu=J_{\mathrm{T}}(1)$.

Let $\{\mathfrak{F}, \mathfrak{F}\}$ be the space of continuous linear mappings of $\mathfrak{H C}$ into itself, with the weak topology. We can apply the above definition to maps of $\Xi$ in to $\{\mathfrak{C}, \mathfrak{C}\}$, and thus define operator-valued integrals.

The standard properties of the integrals hold. In particular:

Property i. Let $\mathfrak{H}_{1}, \mathfrak{H}_{2}$ be complete, locally convex, topological vector spaces; let $\Lambda$ be a continuous linear map of $\mathfrak{H}_{1}$ into $\mathfrak{H}_{2}$, and let $\mathrm{T}$ be an integrable map of $\Xi$ into $\mathfrak{H}_{1}$. Then $\Lambda \cdot T$ is integrable and

$$
\int \Lambda \mathrm{T} d \mu=\Lambda \int \mathrm{T} d \mu
$$

Property $i$ is the analog of Fubini's theorem for vector-valued integrals, and may be also used to justify interchanging orders of differentiation and integration.

Property ii. Let $\mathrm{T}$ be a continuous map of $\Xi$ into $\mathcal{T}$ and let \|\|$_{j}$ be a set of continuous semi-norms on $\mathscr{H}$ which defines the topology of $H$. If $\int\|\mathbf{T}(x)\|_{j} d \mu(x)<\infty$ for all $j$, then $\mathbf{T}$ is integrable.

When applying Property ii to operator-valued integrals, we conclude: Let $\mathrm{T}$ be a continuous map of $\Xi$ into $\{\mathfrak{F}, \mathcal{H}\}$. Suppose that, for each $a \in \mathcal{H}$, $a^{\prime} \in \mathcal{F}^{\prime}$, we have $\int\left|a^{\prime} \cdot(\mathrm{T}(x))(a)\right| d \mu(x)<\infty$. Then $\mathrm{T}$ is integrable.

We shall also want to use the Lie algebra \&S of $G$.

Let $\xi$ be any element of the Lie algebra $B$ of $G$ and $\tau$ any real number. Then $\exp (\tau \xi)$ is well defined and varies for each $\xi \in(\xi)$ over a one-parameter subgroup of $G$. Let $g \rightarrow \mathfrak{U}(g)$ be any (strongly continuous) unitary representation of $G$ on some Hilbert-space. Then $\mathfrak{U}(\exp (\tau \xi))$ is a one-parameter group of unitary operators, hence by Stone's theorem of the form

$$
\mathfrak{u}(\exp (\tau \xi))=\exp [\tau \cdot \mathfrak{u}(\xi)]
$$

such that $\mathfrak{u}(\xi)$ is a uniquely defined skew-adjoint operator. We define in the 
usual manner the left-derivative $L_{\xi}$ in the direction $\xi$ by

$$
\left(L_{\xi}\right)(g)=\lim _{\tau \rightarrow 0} \tau^{-1}\{f([\exp (\tau \xi)] g)-f(g)\}
$$

and a right-derivative $R_{\xi}$ by

$$
\left(R_{\xi} f\right)(g)=\lim _{\tau \rightarrow 0} \tau^{-1}\{f(g \cdot[\exp (\tau \xi)])-f(g)\} .
$$

If $f(g)$ is differentiable at $g$ in the sense of the real analytic structure on $G$, these derivatives exist. Now let $\xi$ be any (noncommutative) polynomial with complex coefficients in the elements of $G$

$$
\xi=\sum c_{i j} \ldots \xi_{i} \xi_{j} \cdots, \quad \xi_{j} \in \oiint .
$$

We put

$$
L_{\xi}=\sum c_{i j} \ldots L_{\xi_{i}} \cdot L_{\xi_{j}} \ldots, \quad R_{\xi}=\sum c_{i j} \ldots R_{\xi_{i}} \cdot R_{\xi_{j}} \ldots
$$

Then

$$
\mathfrak{u}(\xi)=\sum c_{i j} \ldots U\left(\xi_{i}\right) U\left(\xi_{j}\right) \cdots .
$$

is a densely defined operator on the given Hilbert-space. We shall need the following lemma, whose proof is standard and therefore omitted.

LEMMA 1.5. Let $\xi$ be any homogeneous (noncommutative) polynomial (1.20) in the elements of the Lie algebra $\&$ of $G$ and $f(g)$ a sufficiently differentiable complex-valued function on $G$ of compact support. Then

$$
\int_{G}\left(L_{\xi} f\right)(g) \mathfrak{u}(g) d g= \pm \mathfrak{u}(\xi) \int_{G} f(g) \mathfrak{u}(g) d g
$$

and

$$
\int_{G}\left(R_{\xi} f\right)(g) \mathfrak{U}(g) d g= \pm \int_{G} f(g) \mathfrak{U}(g) d g \cdot \mathfrak{U}(\xi) .
$$

The right sides of these two equations exist in the usual sense of the product of a bounded everywhere defined operator by a densely defined operator on Hilbert space.

2. The analogue of the theorem of Paley and Wiener for spherical functions of type $m, n$. In $\S 6$ of Part I we characterized the Fourier transform of the class of indefinitely differentiable spherical functions $f(g)$ of type 0,0 and compact support. We shall now do this for arbitrary type $m, n$. But we shall first give another proof of Theorem 8 of Part I which leads to an improvement of the Corollary to Theorem 8 of Part I.

Since $G / K$ is naturally (analytically) homeomorphic with the upper halfplane of complex numbers $z=x+i y, y>0$, we see that the class of all (continuous, differentiable, etc.) functions $f(g)=f(g k)$ is in natural 1-1 correspond- 
ence with the class of all (continuous, differentiable, etc.) functions $f(x, y)$ defined for $y>0$. The Haar-measure on $G / K$ must (by its uniqueness) go over into the $G$-invariant measure $d x d y / y^{2}$ (up to a multiplicative constant). The spherical functions $f(g)=f\left(k g k^{\prime}\right)$ are in natural 1-1 correspondence with the functions $f(x, y)$ defined for $y>0$ which depend only on the hyperbolic distance of $z=x+i y$ from the point $i$. One can choose the arbitrary multiplicative constant so that

$$
F_{00}(s)=\int_{G} f(g) \phi(g, s) d g=\int_{0}^{\infty} \int_{-\infty}^{\infty} f(x, y) y^{s} \frac{d x d y}{y^{2}}
$$

holds for all (integrable) spherical functions of type 0,0 . Introducing the variable $\tau=y^{-1} \cdot|z-i|^{2}$ and writing $f_{1}(\tau)=f(x, y)$ one obtains for the function $F_{00}(s)$ of $\$ 1$ of Part I

$$
F_{00}(s)=\int_{-\infty}^{\infty} e^{(s-1 / 2) u} q(u) d u
$$

where

$$
q(u)=\tilde{f}\left(e^{u}+e^{-u}-2\right)
$$

and

$$
\tilde{f}(w)=\int_{w}^{\infty}(\tau-w)^{-1 / 2} f_{1}(\tau) d \tau=2 \int_{0}^{\infty} f_{1}\left(w+\tau^{2}\right) d \tau .
$$

These formulas are known and have been used by A. Selberg in his lectures at the Institute for Advanced Study 1954-1955; see also Harish-Chandra [1].

As $f(g)$ varies over all indefinitely differentiable spherical functions of compact support and type 0,0 , i.e. over the space $D_{00}, f_{1}(\tau)$ varies over all indefinitely differentiable functions of compact support of one real variable $\tau \geqq 0$ which are also indefinitely differentiable from the right at $\tau=0$. Hence $\tilde{f}(w)$ varies over certain indefinitely differentiable functions of compact support of one real variable $w \geqq 0$ which are indefinitely differentiable from the right at $w=0$. However, the mapping $f_{1}(\tau) \rightarrow \tilde{f}(w)$ can be inverted by

$$
f_{1}(\tau)=-\frac{1}{\pi} \int_{\tau}^{\infty}(w-\tau)^{-1 / 2} \frac{d \tilde{f}}{d w} d w
$$

which shows that the mapping $f(g) \rightarrow \tilde{f}(w)$ is a topological isomorphism of $D_{00}$ onto the topological vector space of all indefinitely differentiable (including at $w=0+$ ) functions $\tilde{f}(w)$ of compact support and defined for $w \geqq 0$. Hence we obtain all even indefinitely differentiable functions $q(u)$ of compact support. Now we can apply the classical theorem of Paley-Wiener-Schwartz and obtain all entire functions $F_{00}(s)=F_{00}(1-s)$ of exponential type which are rapidly decreasing along $R s=1 / 2$. This gives another proof of Theorem 8 of Part I. 
Now assume that $f_{1}(\tau)=0$ for $\tau>\tau_{0}>0$. Then the above expression for $\tilde{f}(w)$ in terms of $f_{1}(\tau)$ implies immediately that $\tilde{f}(w)=0$ for $w>\tau_{0}$. Conversely, if $\tilde{f}(w)=0$ for $w>\tau_{0}$, then so is $d \tilde{f} / d w$ and hence also $f_{1}(\tau)=0$ for $\tau>\tau_{0}$. The relationship between $\tau$ and the parameter $\zeta$ which we used throughout Part I (see 1.9 ) of $\$ 1$ of Part I) is easily obtained by letting $g_{5}$ act in the upper halfplane, $g_{5}: z \rightarrow e^{25} z$, in particular $g_{5}: i \rightarrow e^{25} i$. Hence

$$
\tau=\frac{\left|e^{25} i-i\right|^{2}}{e^{25}}=e^{25}+e^{-2 \zeta}-2 .
$$

Thus, using the parameterization $f(g) \leftrightarrow f(\zeta)$ as described by (1.10) of $\S 1$ of Part I, we see that $f(\zeta)=0$ for $\zeta>\zeta_{0}>0$ if and only if $q(u)=0$ for $u>2 \zeta_{0}$. Now let us apply the known relationship between the support of $q(u)$ and the exponential type of $F_{00}(s)$ as contained in the classical theorem of Paley and Wiener and we conclude that $F_{00}(s)$ is of exponential type $\leqq 2 \zeta_{0}$ if and only if $q(u)=0$ for $u>2 \zeta_{0}$, which in turn is equivalent to $f(\zeta)=0$ for $\zeta>\zeta_{0}$. This proves

Proposition 2.0. Let $f(g) \in D_{00}$ and write $f(g)=f(\zeta)$ in the sense of (1.10) of $\$ 1$ of Part I. Then $F_{00}(s)$ is an entire function (rapidly decreasing along $R s=1 / 2$ ) of exponential type $\leqq 2 \zeta_{0}$ if and only if $f(\zeta)=0$ for $\zeta>\zeta_{0}$.

By $D$ we denote the space of indefinitely differentiable functions of compact support on $G$. The topology of $D$ can be described as follows: For any compact subset $C$ of $G$, denote by $D_{C}$ the space of all functions of $D$ which vanish outside $C ; D_{C}$ is a metrizable topological vector space, and a sequence $\left\{f_{i}\right\}$ converges to zero in $D_{C}$ if, for $\partial$ any differential operator on $G$ with indefinitely differentiable coefficients, $\partial f_{i} \rightarrow 0$ uniformly on $C$. Among all locally convex topologies that can be put on the set of functions of $D, D$ is given the strongest topology so that the topology induced on each $D_{C}$ is the topology of $D_{c}$. Thus, a fundamental system of neighborhoods of zero in $D$ consists of those convex sets $N$ for which $N \cap D_{C}$ is a neighborhood of zero in $D_{C}$ for all $C$. $D$ is a complete locally convex topological vector space which is not metrizable. $D$ is also a Montel space, that is, the closed bounded subsets of $D$ are compact; moreover, $D$ is reflexive.

An important step in the study of the Fourier transform on $G$ is to describe completely the Fourier transform of the space $D$. The analogous problem for the real line in place of $G$ is solved by means of a theorem of Paley and Wiener (see Paley and Wiener [1], Schwartz [4]); the characterization of the Fourier transform of the spherical functions of type $0,0 \in D$ was given by the authors in Part I of this series (see Ehrenpreis and Mautner [1]). In order to do this for the whole space $D$ we study first the Fourier-transform of $D_{m n}$ for fixed $m$ and $n$.

Let $f(g)$ be any locally summable function on $G$ and put 


$$
\left(P_{m n} f\right)(g)=\int_{K} \int_{K} \bar{\chi}_{n}(k) \bar{\chi}_{m}\left(k^{\prime}\right) f\left(k g k^{\prime}\right) d k d k^{\prime}
$$

where $K$ is again the subgroup of plane rotations of $G, \bar{\chi}_{m}(k)=e^{2 \pi i m \theta}$ if $k$ is the rotation through the angle $\theta$, and $d k$ is the Haar-measure on $K$ normalized so that $\int_{K} d k=1$. From (2.1) one gets at once

$$
\left(P_{m n} f\right)\left(k g k^{\prime}\right)=\chi_{m}(k) f(g) \chi_{n}\left(k^{\prime}\right)
$$

for any $k, k^{\prime} \in K$ and $g \in G$. Now put

$$
D_{m n}=P_{m n} D .
$$

Lemma 2.1. $P_{m n}$ is a continuous projection of $D$. Hence $D_{m n}$ is a closed linear subspace of $D$. It consists exactly of those $f \in D$ which are spherical of type $m, n$ in the sense of (0.1) above.

Proof. It is easily seen that $\left(f, k, k^{\prime}\right) \rightarrow \bar{\chi}_{m}(k) \bar{\chi}_{n}\left(k^{\prime}\right) f\left(k g k^{\prime}\right)$ is a continuous map of $D \times K \times K \rightarrow D$. Since $K$ is compact, the result follows immediately from the theory of integration of vector-valued maps (see $\$ 1$ above).

By Lemma 2.1, we may give $D_{m n}$ the topology induced by $D$ and obtain a complete topological vector space, which we again denote by $D_{m n}$, which is easily seen to be a reflexive Montel space and of type $(L F)$ in the sense of Dieudonné and Schwartz [1].

Lemma 2.2. Let $B$ be a bounded subset of $D$. Then for any $j>0, l>0$ and any $f \in B$ we have

$$
\left(P_{m n} f\right)(g)=0\left[(|m|+1)^{-l}(|n|+1)^{-i}\right]
$$

uniformly for $f \in \Theta$ and $g \in G$. In particular, for any $f \in D, P_{m n} f$ is rapidly decreasing in $m$ and $n$ (see Schwartz [4]).

Proof. By (1.9) of Part I or (1.0) of the present Part II, we can write $g=k g_{5} k^{\prime}$. Let $\vartheta$ be an element of the Lie algebra of $K$, then the left-derivative $L_{\vartheta}$ and the right-derivative $R_{\vartheta}$ have been defined in $\S 1$ (see (1.18) and (1.19) above). Now, since $B$ is a bounded subset of $D$, it follows that for any positive integers $j$ and $l\left(L_{v}^{j} R_{v}^{l} f\right)(g)$ is bounded uniformly for $g \in G, f \in D$. Thus

$$
\sup _{s \in ß, \bullet \in G}\left|\left(L_{v}^{j} R_{\vartheta}^{l} f\right)(g)\right| \leqq M_{l, j} .
$$

Then

$$
\begin{aligned}
\left(P_{m n} L_{\vartheta}^{j} R_{\vartheta}^{l} f\right)(g) & =\iint_{K \times K} \bar{\chi}_{m}(k) \bar{\chi}_{n}\left(k^{\prime}\right)\left(L_{\vartheta}^{j} R_{\vartheta}^{l} f\right)\left(k g k^{\prime}\right) d k d k^{\prime} \\
& =(i m)^{l}(i n)^{j} \iint_{K \times K} \bar{\chi}_{m}(k) \bar{\chi}_{n}\left(k^{\prime}\right)\left(L_{\vartheta}^{j} R_{\vartheta}^{l} f\right)\left(k g k^{\prime}\right) d k d k^{\prime}
\end{aligned}
$$


by integration by parts. Thus

$$
P_{m n} L_{\vartheta}^{i} R_{\vartheta}^{l} f=(i m)^{l}(i n)^{j} P_{m n} f .
$$

Since $B$ is bounded, all the functions $\in B$ have their supports in a fixed compact subset $\mathcal{C}$ of $G$. It follows immediately from (2.6) that

$$
\sup _{\bullet \in G, f \in B}\left|P_{m n}\left(L_{\vartheta}^{j} R_{\vartheta}^{l} f\right)(g)\right| \leqq M_{l, j} \cdot \mu(\mathbb{C})
$$

where $\mu(\mathcal{C})$ is the Haar-measure of $\mathcal{C}$. By (2.7) this means

$$
\sup _{o \in G, f \in \mathbb{B}}|m|{ }^{l}|n|^{i}\left|\left(P_{m n} f\right)(g)\right| \leqq M_{l, j} \mu(\mathbb{e}) .
$$

This proves the assertion of Lemma 2.2.

For future reference we state the following lemma whose proof is contained in the above.

Lемма 2.3. For all positive integers $j, l$, all integers $m, n$ and any $f \in D, g \in G$

$$
P_{m n} L_{\vartheta}^{j} R_{\vartheta}^{l} f=(i m)^{j}(i n)^{l} P_{m n} f .
$$

Let us denote by $\{D, D\}$ the space of continuous linear maps of $D$ into $D$ with the compact-open topology, that is, $\Re$ is a neighborhood of zero in $\{D, D\}$ if we can find a compact set $B$ of $D$ and a neighborhood of zero $\mathfrak{T}$ in $D$ so that $\mathscr{N}$ contains all $s \in\{D, D\}$ for which $s(f) \in \mathscr{N}$ for any $f \in ß$. It is readily verified that $\{D, D\}$ is a complete, Hausdorff, locally convex, topological vector space. By $I$ we denote the identity map of $D \rightarrow D$.

Lemma 2.4. The series $\sum_{m, n} P_{m n}$ converges in the topology of $\{D, D\}$ to $I$.

Proof. Let $B$ be a compact set in $D$; we must first prove that $\sum P_{m n} f$ converges in the topology of $D$ uniformly for $f \in ß$. Now (see Dieudonné and Schwartz [1]) all functions $f \in ß$ have their supports in a fixed compact set $\mathfrak{C}^{*}$ of $G$; since $K$ is compact it follows easily that we can find a compact set $\mathcal{C}$ of $G$ so that, for all $m, n$ and all $f \in ß, P_{m n}(f) \in D_{\mathbb{C}}$. We shall show that the series $\sum P_{m n} f$ converges uniformly in $D_{C}$ for $f \in \Theta$. For this purpose, it is sufficient to show that, for $\partial$ any operator of partial differentiation on $G$ with indefinitely differentiable coefficients,

$$
\sum \max _{o \in C}\left|\left(\partial P_{m n}(f)\right)(g)\right|
$$

converges uniformly for $f \in ß$. For any $m, n$, and any $k, k^{\prime} \in K$, let $t_{m n}\left(k, k^{\prime}\right)$ be the map of $D \rightarrow D$ :

$$
\left(\left(t_{m n}\left(k, k^{\prime}\right)\right) f\right)(g)=\bar{\chi}_{m}(\bar{k}) \bar{\chi}_{n}\left(k^{\prime}\right) f\left(k g k^{\prime}\right)
$$

for any $f \in D, g \in G$. Then it follows from the theory of integration of vector 
valued maps (see $\$ 1$ above) that, for any $f \in D$,

$$
\left(\partial P_{m n} f\right)=\int_{K} \int_{K} \partial\left(t_{m n}\left(k, k^{\prime}\right)\right)(f) d k d k^{\prime} .
$$

Then, by the above,

$$
\begin{aligned}
\partial P_{m n} f & =\int_{K} \int_{K} \partial\left[t_{m n}\left(k, k^{\prime}\right)\right](f) d k d k^{\prime} \\
& =\int_{K} \int_{K} \bar{\chi}_{m}(k) \bar{\chi}_{n}\left(k^{\prime}\right) \partial\left[L\left(k^{-1}\right) R\left(k^{\prime}\right)\right](f) d k d k^{\prime}
\end{aligned}
$$

where $L(k)$ denotes left translation by $k$ and $R\left(k^{\prime}\right)$ denotes right translation by $k^{\prime}$. Since $\left\{\partial L\left(k^{-1}\right) R\left(k^{\prime}\right) f\right\}_{k, k^{\prime} \in K, f \in \beta}$ is bounded in $D$, it follows, as in the proof of Lemma 2.1, that

$$
\left|\left(\partial P_{m n} f\right)(g)\right| \leqq \alpha(1+|m|)^{-2}(1+|n|)^{-2}
$$

where $\alpha$ is a constant depending only on $\partial$ and $B$. Thus,

$$
\sum \max _{g \in C}\left|\left(\partial P_{m n} f\right)(g)\right|
$$

converges uniformly for $f \in \beta$ which proves the convergence of $\sum P_{m n}$ in $\{D, D\}$.

We must still show that $\sum P_{m n}$ converges to $I$. For this it is sufficient to show that, for any $f \in D$, and any $g \in G, \sum\left(P_{m n} f\right)(g)=f(g)$. Now, consider the function $h$ on $K \times K: h\left(k, k^{\prime}\right)=f\left(k g k^{\prime}\right)$. Since $K$ is the ordinary circle group, we have

$$
h\left(k, k^{\prime}\right)=\sum_{m, n} \chi_{m}(k) \chi_{n}\left(k^{\prime}\right) h_{m n}
$$

where

$$
\begin{aligned}
h_{m n} & =\int_{K} \int_{K} h\left(k, k^{\prime}\right) \bar{\chi}_{m}(k) \bar{\chi}_{n}\left(k^{\prime}\right) d k d k^{\prime} \\
& =\left(P_{m n} f\right)(g)
\end{aligned}
$$

because we have already shown that the right side of 2.9 converges absolutely and uniformly for $\left(k, k^{\prime}\right) \in K \times K$. Taking $\left(k, k^{\prime}\right)=(1,1)$ in $(2.8)$ we find

$$
f(g)=h(1,1)=\sum_{m, n}\left(P_{m n} f\right)(g)
$$

which is the desired result.

The Fourier transform on $G$ is given as follows. Let $f(g)$ be a complexvalued integrable function of compact support on $G$. Then we know that the integral 


$$
F_{m n}(s)=\int_{G} f(g) u_{m n}(g, s) d g
$$

exists for all integers $m, n$ and every complex number $s$. Now let $v_{m n}^{ \pm}(g, l)$ be the matrix-coefficients of the discrete series of irreducible unitary representations of $G$ (cf. (1.5)-(1.8) or $\$ 1$ above). We put

$$
f_{m n}^{ \pm}(l)=\int_{G} f(g) v_{m n}^{ \pm}(g, l) d g .
$$

The Fourier transform of $f(g)$ is the triple $\left\{F_{m n}(s), f_{m n}^{+}(l), f_{m n}^{-}(l)\right\}$ and we write $\left({ }^{3}\right)$

$$
\begin{aligned}
\mathfrak{T} f & =\left\{F_{m n}(s), \hat{f_{m n}^{+}}(l), \overline{f_{m n}}(l)\right\}, \\
\mathfrak{T}_{0} f & =\left(F_{m n}(s)\right) .
\end{aligned}
$$

We shall now prove the analogue of the Paley-Wiener theorem for the space $D_{m n}$ for fixed $m$ and $n$, i.e. characterize $T D_{m n}$.

THEOREM 2.1. Let $m$ and $n$ be any fixed integers. The following two classes of complex valued functions $F(s)$ are identical:

(a) The class of all functions of $s$ which are of the form $\int f(g) u_{m n}(g, s) d g$ with $f(g)$ varying over $D_{m n}$ for fixed $m, n$.

(b) The class of all entire functions $F(s)$ of exponential type which are rapidly decreasing on the line $R s=1 / 2$ and satisfy

$$
F(1-s)=\chi_{m n}(s) F(s)
$$

and

$$
F(s)=0\left\{\begin{array}{l}
\text { if } m \geqq 0, \quad n<0 \text { for } s=0,1, \cdots, m, \\
\text { if } m<0, \quad n \geqq 0 \text { for } s=0,-1,-2, \cdots, 1-n . \\
\text { if } m \leqq 0, \quad n>0 \text { for } s=0,1,2, \cdots, m, \\
\text { if } m>0, \quad n \leqq 0 \text { for } s=0,-1,-2, \cdots, n+1
\end{array}\right.
$$

REMARK. The functional equation (2.13) implies that

(2.14a) $F(s)=0$ for $s=-|n|+1,-|n|, \cdots,-|m|$ if $|n|>|m|$ and for $s=|n|+1,|n|+2, \cdots,|m|$ if $|m|>|n|$.

Proof. Assume $f(g) \in D$. From the expression (1.4) of Part I for the $u_{m n}(g, s)$ it follows that $u_{m n}(g, s)$ is for fixed $m, n$ and $g$ an entire function of

(3) Note that if $f(g)$ is spherical of type 0,0 then $f_{m n}^{+}(l)=f_{m n}^{-}(l)=0$ for all $m, n$ and $l$ and $F_{m n}(s)=0$ for all $s, m, n$ unless $m, n=0,0$ so that the present definition of $\mathfrak{I} f$ coincides with that of Part I for spherical functions of type 0,0 if we identify the matrix $\left\{F_{m n}(s) \delta_{m 0} \delta_{n 0}\right\}$ with the function $F_{00}(s)$. 
$s$ of exponential type, simultaneously analytic in $g$ and $s$. Also $\left|u_{m n}(g, s)\right|$ $\leqq u_{00}(g, \sigma)=\phi(g, \sigma)$, hence Theorem 1 of Part I implies that $u_{m n}(g, s)$ is of exponential type $\leqq 2$ (see also Lemma 1.1 above) where $g=k g_{5} k^{\prime}$ as in (2.5) above (see also $\S 1$ of Part I). Hence, $f(g)$ being of compact support, the integral $\int f(g) u_{m n}(g, s) d g$ converges to an entire function of exponential type. Moreover, if $f\left(k g_{\zeta} k^{\prime}\right)=0$ for $\zeta>v$ then $F_{m n}(s)$ is of exponential type $\leqq 2 v$. Since $u_{m n}(g, s)$ satisfies the functional equation (2.13), so does $F_{m n}(s)$ (see $\S 3$ of Part I and Bargmann [1, (6.23), (6.25), (6.29) and (10.27)]). Moreover Lemma 1.4 implies (2.14). This proves Theorem 2.1 one way.

To prove the converse we assume $F(s)$ is a given numerical valued entire function of exponential type rapidly decreasing on $R s=1 / 2$ and satisfying (2.13) and (2.14). We proceed in several steps.

Lemma 2.5. Let $f(g) \in L_{2}(G)$ and $P_{m n} f=f$. Assume that 1.i.m. $\int f(g) u_{m n}(g$, $1 / 2+i t) d g=F(1 / 2+i t)$ satisfies $s^{i}(1-s)^{j} F(s) \in L_{2}(R s=1 / 2, d t)$ for every positive integer $j$. Then $f(g)$ is indefinitely differentiable.

Proof. Let $\Delta$ be again the group-invariant Laplace operator as at the beginning of $\S 4$ of Part I. If $f(g) \in D$, then $\mathfrak{T} \Delta f=\left\{s(1-s) F_{m n}(s), l(1-l) f_{m n}^{+}(l)\right.$, $\left.l(1-l) f_{m n}^{-}(l)\right\}$. For fixed $m, n$ the restriction of $\Delta$ to $D_{m n}$ is an ordinary formally self-adjoint second order differential operator $\Delta_{m n}$ (see Bargmann [1, $\S 10 \mathrm{c}]$ ). We now define a self-adjoint (hypomaximal) extension $\widetilde{\Delta}_{m n}$ of $\Delta_{m n}$ whose domain is a dense linear subspace of the Hilbert space $P_{m n} L_{2}(G)$ as follows: If $f \in P_{m n} L_{2}(G)$, then $f$ shall be in the domain of $\tilde{\Delta}_{m n}$ if and only if $s(1-s) F_{m n}(s) \in L_{2}(R s=1 / 2, d t)$. We define $\tilde{\Delta}_{m n} f$ by $\mathfrak{T} \tilde{\Delta}_{m n} f=\left\{s(1-s) F_{m n}(s)\right.$, $\left.l(1-l) f_{m n}^{ \pm}(l)\right\}$.

Note that since $P_{m n} f=f, f_{m n}^{ \pm}(l) \neq 0$ only for a finite number of values of $l$. The operator $\widetilde{\Delta}_{m n}$ so defined is self-adjoint hypermaximal densely defined on the Hilbert space $P_{m n} L_{2}(G)$ and $\tilde{\Delta}_{m n} \supset \Delta_{m n}$. By hypothesis $s^{j}(1-s)^{i} F_{m n}(s)$ $\in L_{2}(R s=1 / 2, d t)$ and since $f_{m n}^{ \pm}(l)=0$ for almost all $l$, the Plancherel formula for $L_{2}(G)$ restricted to $P_{m n} L_{2}(G)$ tells us that there exists a unique element $f_{j} \in P_{m n} L_{2}(G)$ such that $\mathfrak{T} f_{j}=\left\{s^{i}(1-s)^{j} F_{m n}(s), l^{j}(1-l)^{i} f_{m n}^{ \pm}(l)\right\}$. Hence by the definition of $\widetilde{\Delta}_{m n}$ we see that $f$ is in the domain of $\tilde{\Delta}_{m n}^{j}$ and $f_{j}=\tilde{\Delta}_{m n}^{j} f$ for every $j=0,1,2, \cdots$. This implies that $f(g)$ is indefinitely differentiable as follows: For $\psi \in D_{m n}$, we have $\left\langle\tilde{\Delta}_{m n}^{j} f, \psi\right\rangle=\left\langle f, \Delta_{m n}^{j} \psi\right\rangle$. Given any integer $r>0$ there exists an integer $j>0$ and a Green's kernel $\Omega_{j}$ of class $C^{r}$ such that $\Omega_{j} \Delta^{j}$ $=\Delta^{i} \Omega_{j}=I$ on $D_{m n}$. Now $f_{j}=\Delta^{i} f \in L_{2}$, hence is the second derivative (in the sense of distributions) of a continuous function, hence $f=\Omega_{j} f_{j}$ is of class $C^{r-2}$. (A similar argument is carried out in detail in L. Schwartz [4].) This proves Lemma 2.5 .

REMARK. Lemma 2.5 can also be proved using the Fourier inversion formula for $G$ by showing that $\Delta$ can be applied under the integral sign. This can be demonstrated e.g. by using the theory of integration of vector-valued maps (see Bourbaki [2]). 
We shall now complete the proof of Theorem 2.1. Let $E$ be the space of all indefinitely differentiable functions on $G$ and $E_{m n}=P_{m n} E$.

We are given a function $F(s)$ belonging to the class (b) of Theorem 2.1 for fixed $m$ and $n$. We conclude first that there exists a unique element $f(g)$ $\in P_{m n} L_{2}(G)$ such that $\mathfrak{T} f=\left\{\mathfrak{F}(s), \mathfrak{f}^{+}(l), \mathfrak{f}^{-}(l)\right\}$ where the operator $\mathfrak{F}(s)$ has for each complex $s$ the matrix coefficients $F_{\mu \nu}(s)=\delta_{\mu m} \delta_{\nu n} F(s)$ and the operator $\mathrm{f}^{ \pm}(l)$ has for each integer $l>0$ the matrix-coefficients $f_{\mu \nu}^{ \pm}(l)=\delta_{\mu m} \delta_{\nu n} F(-l) \omega_{m n}(l)$. Thus $\mathrm{f}^{ \pm}(l)$ has for each $l$ only one nonvanishing matrix coefficient $m, n$ and for this $m, n$ the function $f_{m n}^{ \pm}(l)$ vanishes for almost all $l$, as follows from the definitions. Also $F(1 / 2+i t) \in L_{2}(R s=1 / 2, \delta t)$. Hence using (2.3), the Plancherel formula for $G$ (and $\S 3$ of Mautner [4]) we see that $\left\{\mathfrak{F}(s), \mathfrak{f}^{+}(l), \mathfrak{f}^{-}(l)\right\}$ are in the range of $\mathfrak{I}$ where at the moment $\mathfrak{I}$ is considered to be a unitary mapping defined for the whole of $L_{2}(G)$ (cf. Mautner [4]). Thus there exists an element $f(g) \in P_{m n} L_{2}(G)$ such that $\mathfrak{T} f=\left\{\mathfrak{F}(s), \mathfrak{f}^{+}(l), \mathfrak{f}^{-}(l)\right\}$. By Lemma 2.5, $f(g) \in E$, hence $f(g) \in E_{m n}$.

By Bargmann $\left[1\right.$, p. 613, formulae (6.22)] there exists an element $\xi_{1}$ in the associative enveloping algebra such that the operator $U\left(\xi_{1}, 1 / 2+i t\right)$ as defined in $\$ 1$ (see (1.22) above) satisfies

$$
U\left(\xi_{1}, 1 / 2+i t\right) e^{2 \pi i M \theta}=(M-1 / 2-i t) e^{2 \pi i(M-1) \theta} .
$$

Thus the element $\xi_{1}^{m}$ satisfies

$$
\begin{aligned}
U\left(\xi_{1}^{m}, s\right) e^{2 \pi i M \theta} & =\prod_{j=1}^{m}(M-j+1-s) e^{2 \pi i(M-m) \theta} \text { for } R s=1 / 2 \text { and } m>0 .
\end{aligned}
$$

Similarly there exists an element $\xi_{2}$ such that

$$
\begin{aligned}
U\left(\xi_{2}^{n}, s\right) e^{2 \pi i N \theta} & =\prod_{j=1}^{n}(N+j-1+s) e^{2 \pi i(N+n) \theta} \text { for } R s=1 / 2 \text { and } n>0 .
\end{aligned}
$$

Now let $h(g) \in D$ and again $\mathfrak{H}(s)=\int h(g) U(g, s) d g$. Put $a(g)=\left(L_{\xi_{1}^{m}}^{m} h\right)(g), b(g)$ $=\left(R_{\xi_{2}^{n}}^{n} a\right)(g), c(g)=\left(R_{\xi_{1}^{m}}^{m} h\right)(g)$ and $e(g)=\left(L_{\xi_{1}^{n}}^{n} c\right)(g)$. We write $\mathfrak{A}(s)=\int a(g) U(g$, $s) d g, \mathbb{B}(s)=\int b(g) U(g, s) d g$ etc. Then Lemma 1.4 implies for $R s=1 / 2$

$$
\mathfrak{A}(s)=U\left(\xi_{1}^{m}, s\right) \mathfrak{H}(s), \quad \mathbb{B}(s)=\mathfrak{A}(s) U\left(\xi_{2}^{n}, s\right) \text { etc. }
$$

Hence, combining these formulas we get for the matrix coefficients $A_{p q}(s)$, $B_{p q}(s), \cdots$ of the operators $\mathfrak{A}(s), \mathbb{B}(s), \cdots$

$$
\begin{aligned}
& A_{p q}(s)=\prod_{j=1}^{m}(p-j+1-s) H_{p-m, q}(s), \\
& B_{p q}(s)=\prod_{j=1}^{n}(q-n+j-1+s) A_{p, q-n}(s) .
\end{aligned}
$$


Hence

$$
B_{p q}(s)=\prod_{j=1}^{m}(p-j+1-s) \prod_{j=1}^{n}(q-j+s) H_{p-m, q-n}(s) .
$$

Similarly

$$
C_{p q}(s)=\prod_{j=1}^{m}(q+j-s) H_{p, q+m}(s)
$$

hence

$$
E_{p q}(s)=\prod_{j=1}^{m}(q+j-s) \prod_{j=1}^{n}(p-j+1-s) H_{p-n, q+m}(s) .
$$

These formulas hold for $R s=1 / 2$ by Lemma 1.4. However since $h(g) \in D$, so are $a, b, c$ and $e$, therefore $A_{p q}(s), B_{p q}(s), \cdots$ are all entire functions of $s$. Hence $(2.20), \cdots,(2.23)$ hold for every complex number $s$. We have thus proved

Lemma 2.6. Let $h(g) \in D$. For any non-negative integers $m$ and $n$ put a $(g)$ $=\left(L_{\xi_{1}}^{m} h\right)(g), \quad b(g)=\left(R_{\xi_{2}}^{n} a\right)(g), \quad c(g)=\left(R_{\xi_{1}}^{m} h\right)(g) \quad$ and $\quad e(g)=\left(L_{\zeta_{1}}^{n} c\right)(g)$. Then $H_{p q}(s), A_{p q}(s), \cdots$ are related by (2.19), $\cdots,(2.22)$ for every complex number $s$ and all integers $p, q$.

We shall assume at first that $m, n \geqq 0$ and $n \geqq m$. Suppose first that $f(g)$ is such that

$$
F(s)=0 \text { for } s=0-1,-2, \cdots,-m .
$$

Write

$$
\begin{aligned}
\eta_{m n}(s) & =\prod_{j=1}^{m}(j-s)(j-1+s) \prod_{j=m+1}^{m}(j-s) \\
& =\prod_{j=1}^{n}(j-s) \prod_{j=1}^{m}(j-1+s)
\end{aligned}
$$

so that $F(s) / \eta_{m n}(s)=F(1-s) / \eta_{m n}(1-s)$. Thus, by the known result for $D_{00}$ (see Proposition 2.0 above or Theorem 8 of Part I) there is an $h(g) \in D_{00}$ with

$$
\int h(g) u_{00}(g, s) d g=F(s) / \eta_{m n}(s) .
$$

As in Lemma 2.6, call $b=R_{\xi_{2}^{n}} L_{\xi_{1}^{m}} h$. Then it is clear that $b(g) \in D$, and, by Lemma 2.6 we even have $b(g) \in D_{m n}$. In addition, $b(g)=f(g)$ because both have the same Fourier transform $F(s)$. This concludes the proof for the case $n \geqq m \geqq 0$ as regards functions $F(s)$ satisfying (2.24).

Let us note the following: $D$ is dense in $L_{2}(G)$ so, by applying $P_{m n}$, we 
see that $D_{m n}$ is dense in $P_{m n} L_{2}(G)$. The matrix coefficients $u_{m n}(g, l)(l=0,-1$, $-2, \cdots,-m)$ are real analytic linearly independent functions on $G$. Therefore, they are linearly independent over an arbitrarily small open subset of $G$. Thus we can find indefinitely differentiable functions $q_{0}\left(g_{\xi}\right), \cdots, q_{m-1}\left(g_{\xi}\right)$ of arbitrarily small supports in $\zeta$, so that

$$
\int q_{i}(g) u_{m n}(g, j) d g=\delta_{i j}, \quad i, j=0,1, \cdots, m-1 .
$$

We write $Q_{i}(s)=\int q_{i}(g) u_{m n}(g, s) d g$; since $q_{i}(g) \in D_{m n}$, each $Q_{i}(s)$ is an entire function of exponential type which is rapidly decreasing on $R s=1 / 2$. The exponential types of the $Q_{i}(s)$ may be made as small as we want.

For an arbitrary $F(s)$ as above, call

$$
\tilde{F}(s)=F(s)-\sum_{i=0}^{m-1} F(-i) Q_{i}(s) .
$$

Then there exists $\tilde{f}(g) \in E_{m n} \cap L_{2}$ satisfying $\mathfrak{I}_{0} \tilde{f}=\tilde{F}(s)$. Also $\tilde{F}(s)$ satisfies (2.24), so, by what was proven above, $\tilde{f}$ is of compact support, i.e. $\tilde{f} \in D_{m n}$. But, $f(g)=\tilde{f}(g)+\sum_{i=0}^{m-1} F(-i) q_{i}(g)$ because both members of this equation have the same Fourier transform. This proves that $f(g) \in D_{m n}$. This completes the proof of Theorem 2.1 for the case $n \geqq m \geqq 0$. The cases $m \geqq m \geqq 0$ and $m, n \leqq 0$ can be handled similarly.

To complete the proof of Theorem 2.1 , we consider the case $m \geqq 0, n<0$, $-n \geqq m$; the other remaining cases are handled similarly. Suppose $F(s)$ belongs to the class (b); then it follows immediately from (2.15a) and $(2.15 \mathrm{~b})$ that $F(s)=0$ for $s=n+1, \cdots, 0,1, \cdots, m$. Thus, if we set

$$
\eta_{m n}(s)=\prod_{j=1}^{m}(j-1+s) \prod_{j=1}^{-n}(j-s),
$$

we have, as before, $F(s)=\mathfrak{I}_{0} L_{\xi_{1}^{m}} \cdot R_{\xi_{1}^{|| \mid}} h$ where $h \in D_{00}$ is defined by

$$
H(s)=F(s) / \eta_{m n}(s) .
$$

Since $L_{\xi_{1}^{m}}^{m} R_{\xi_{1}}^{|n|} h \in D_{m n}$, this concludes the proof of Theorem 2.1.

PROPOSITION 2.1. $f(g)=f\left(k g_{\zeta} k^{\prime}\right) \in D_{m n}$ vanishes for $\zeta \geqq v$ if and only if $F(s)$ is an entire function of exponential type $\leqq 2 v$, i.e. $v$ is the smallest number such that $f\left(g_{\zeta}\right)=0$ for all $\zeta \geqq v$, if and only if $2 v$ is exactly the exponential type of $F(s)$.

Proof. If $(m, n)=(0,0)$, the result is proved above (see Proposition 2.0). It follows immediately from the Lemma 1.1 that, for arbitrary $(m, n)$, if $f(g)=0$ for $\zeta \geqq v$ then $F(s)$ is of exponential type $\leqq 2 v$. Let $(m, n)$ be arbitrary. Then, from the known result for $(0,0)$ and the fact that, if $h(g) \in D_{00}$ vanishes for $\zeta \geqq v$ then also $P_{m 0} \xi_{1}^{m} * h(g) * P_{0 n} \xi_{2}^{n}$ vanishes for $\zeta \geqq v$, we deduce as in the proof of Theorem 2.1 that if $F(s)$ is of exponential type $\leqq 2 v$, and satisfies 
(2.24), then $f(g)=0$ for $\zeta \geqq v$. Finally, we note that if $F(s)$ does not satisfy (2.24), then using the above result for functions satisfying (2.24) and the fact that the functions $q_{i}\left(g_{5}\right)$ introduced in the proof of Theorem 2.1 may be assumed to have their supports in an arbitrary small neighborhood of 0 , we deduce easily the general result.

We have seen in $\$ 1$ above (Lemma 1.1) that $u_{m n}\left(g_{5}, s\right)$ is an entire function of $s$ of exponential type $\leqq 2 \zeta$. As an immediate consequence of Theorem 2.1 and Proposition 2.1 we have

COROLlaRY 1. For each $\zeta>0$ and all integers $m, n$ the function $u_{m n}\left(g_{5}, s\right)$ is an entire function of $s$ of exponential type precisely $2 \zeta$.

As another immediate consequence of Proposition 2.1 and the well known property of the exponential type of a product we have

Corollary 2. Let $f_{1}(g) \in D_{m n}$ and $f_{2}(g) \in D_{n p}$. Suppose $\zeta_{j}$ is the smallest number such that $f_{j}\left(g_{\zeta}\right)=0$ for all $\zeta>\zeta_{j}$. Then $\zeta_{1}+\zeta_{2}$ is the smallest number such that $\left(f_{1} * f_{2}\right)\left(g_{\zeta}\right)=0$ for all $\zeta>\zeta_{1}+\zeta_{2}$.

Theorem 2.1 characterizes completely the set of Fourier transforms of the functions of $D_{m n}$. Denote by $\mathfrak{T}_{0} D_{m n}$ the set of all $\mathfrak{T}_{0} f$ for $f \in D_{m n}$, so $\mathfrak{I}_{0} D_{m n}$ is a vector space, and $\mathfrak{I}_{0}$ is a one-one linear map of $D_{m n}$ onto $\mathfrak{T}_{0} D_{m n}$. We give $\mathfrak{T}_{0} D_{m n}$ the topology to make $\mathfrak{T}_{0}$ a topological isomorphism, and we obtain a topological vector space which we denote again by $\mathfrak{T}_{0} D_{m n}$. For any $v>0$ we call $D_{m n}^{v}$ the space of those $f \in D_{m n}$ where $f(g)=0$ whenever $g=k_{0} g_{\zeta} k_{\theta}, \zeta \geqq v$. Thus, $D_{m n}$ is the inductive limit of the spaces $D_{m n}^{v}$ for $v$ integral. Denote by $\mathfrak{T}_{0} D_{m n}^{v}$ the space of all $\mathfrak{T}_{0} f, f \in D_{m n}^{v}$. In order to describe the topology of $\mathfrak{T}_{0} D_{m n}$, we shall first describe the topology of $\mathfrak{T}_{0} D_{m n}^{v}$. Since $\mathfrak{T}_{0} D_{m n}^{v}$ is metrizable, it suffices to describe the convergence of sequences.

THEOREM 2.2. Let $\left\{F_{j}\right\}$ be a sequence in $\mathfrak{T}_{0} D_{m n}^{v}$. A necessary and sufficient condition that $F_{j} \rightarrow 0$ in $\mathfrak{T}_{0} D_{m n}^{v}$ is: For any polynomial $P, P F_{j} \rightarrow 0$ uniformly in some strip about $R(s)=1 / 2$.

Proof. For each $j$, set $f_{j}=\mathfrak{T}_{0}^{-1} F_{j}$. Suppose first that $f_{j} \rightarrow 0$ in $D_{m n}^{v}$. Then for any $r, \Delta^{r} f_{j} \rightarrow 0$ uniformly on $G$. From this it follows immediately that

$$
s^{r}(1-s)^{r} F_{j}(s)=\int_{G} \Delta^{r} f_{j}(g) u_{m n}(g, s) d g \rightarrow 0
$$

uniformly on $R(s)=1 / 2$. Thus, for any polynomial $Q, Q f_{j} \rightarrow 0$ uniformly on $R(s)=1 / 2$. By Proposition 2.2 we can find a $v^{\prime}$ such that all $F_{j}$ are of exponential type $\leqq v^{\prime}$, it follows easily from a theorem of Phragmén and Lindelöf that $Q F_{j} \rightarrow 0$ uniformly in any strip about $R(s)=1 / 2$.

Conversely, suppose that, for any polynomial $Q, Q F_{j} \rightarrow 0$ uniformly in some (hence any) strip about $R(s)=1 / 2$. For each $j$, if $\min (m, n) \geqq l>0$, set $f_{j}^{+}(l)=\omega_{m n}(l) F_{j}(-l)$, while if $\min (-m,-n) \geqq l>0$, set $f_{j}^{-}(l)=\omega_{m n}(l) F_{j}(-l)$; 
for all other choices of $m, n, l$, set $f_{j}^{+}(l)=0, f_{j}^{-}(l)=0$. It follows that for each $l, f_{j}^{+}(l) \rightarrow 0, f_{j}^{-}(l) \rightarrow 0$.

Now, set $f_{j}=\mathfrak{T}_{0}^{-1} F_{j}$. Then (see Harish-Chandra [1], Mautner [4]) for $s=1 / 2+i t$

$$
\begin{aligned}
f_{j}(g)= & \int_{R(s)=1 / 2} F_{j}(s)\left(u_{m n}\right)^{*}(g, s) \delta t+\sum_{l}(2 l-1) f_{j}^{+}(l)\left(v_{m n}^{+}\right)^{*}(g, l) \\
& +\sum_{l}(2 l-1) \overline{f_{j}}(l)\left(\overline{v_{m n}}\right)^{*}(g, l)
\end{aligned}
$$

where $\delta t=t \tanh \pi t d t, d t$ being the usual Lebesgue measure. Since $\left|u_{m n}(g, s)\right|$ $\leqq 1$ for all $g, R(s)=1 / 2$, we see that

$$
\left|\int_{R(s)=1 / 2} F_{j}(s) u_{m n}(g, s)^{*} d s\right| \rightarrow 0
$$

uniformly in $g$ and even, for any $r$,

$$
\begin{aligned}
\left|\Delta^{r} \int_{R(s)=1 / 2} F_{j}(s)\left(u_{m n}\right)^{*}(g, s) \delta s\right| & \\
= & \left|\int_{r_{(s)=1 / 2}} s^{r}(1-s)^{r} F_{j}(s)\left(u_{m n}\right)^{*}(g, s) \delta s\right| \rightarrow 0
\end{aligned}
$$

uniformly in $g$. On the other hand each $v_{m n}^{+}(g, l) \in L_{\infty}(G)$ since they are the matrix-coefficients of unitary representations. Thus, for any $r$,

$$
\Delta^{r}\left[\sum_{l}(2 l-1) f_{i}^{+}(l)\left(v_{m n}^{+}\right)^{*}(g, l)+\sum_{l}(2 l-1) \tilde{f}_{j}^{-}(l)\left(v_{m n}^{-}\right)^{*}(g, l)\right] \rightarrow 0
$$

uniformly on $G$. This proves that, for any $r, \Delta^{r} f_{j} \rightarrow 0$ uniformly on $G$, which is the desired result.

For any $v>0$ denote by $\widetilde{D}_{m n}^{v}$ the space of all $F(s) \in \mathfrak{T}_{0} D_{m n}$ which are of exponential type $\leqq 2 v$. The topology of $\widetilde{D}_{m n}^{v}$ is defined by the countable number of semi-norms

$$
\sup _{0 \leqq R s \leqq 1}\left|s^{r} F(s)\right|
$$

for $r=0,1,2, \cdots$. Thus $\tilde{D}_{m n}^{v}$ is metrizable. Denote by $\widehat{D}$ the ordinary Fourier transform on the additive group of real numbers of the space $D=D(-\infty, \infty)$ of Schwartz of all indefinitely differentiable functions of compact support defined on the real line, and make the change of variable $s \rightarrow 1 / 2+i s$ (see (0.2) and (0.3) above). The space $\widetilde{D}_{m n}^{v}$ is a closed subspace of $\widehat{D}$, hence $\widetilde{D}_{m n}^{v}$ is complete. Now, by Proposition 2.2 we have $\widetilde{D}_{m n}^{o}=\mathfrak{T}_{0} D_{m n}^{v}$ for every $v>0$. Moreover, by Theorem 2.2 above, the topology induced by 
$\mathfrak{T}_{0} D_{m n}^{v}$ on $\widetilde{D}_{m n}^{v}$ is exactly the topology defined on $\widetilde{D}_{m n}^{2 v}$ by the above seminorms. Thus (see Dieudonné and Schwartz [1]) $\mathfrak{I}_{0} D_{m n}$ is the inductive limit of the space $\widetilde{D}_{m n}^{v}$ for $v$ integral.

For each $v>0$ denote by $\widehat{D}^{v}$ the space of all $F \in \widehat{D}$ of exponential type $\leqq 2 v$, with the topology induced by $\widehat{D}$. We know (see Ehrenpreis $[3 ; 4]$ ) that $\widehat{D}$ is the inductive limit of the spaces $\widehat{D}^{v}$ for $v$ integral. It follows from the definition of the topology of $\widetilde{D}_{m n}^{v}$, that $\widetilde{D}_{m n}^{v}$ has the topology induced by $\widehat{D}$. Thus, by the definition of the topology of an inductive limit (see Dieudonné and Schwartz [1]) it follows that the topology of $\mathfrak{T}_{0} D_{m n}$ is stronger than the topology induced by $D$.

On the other hand, if $\mathfrak{T}$ is a convex neighborhood of zero in $\mathfrak{T}_{0} D_{m m}$, then denote by $\mathfrak{N}$ the set of $F \in \widehat{D}$ for which $[F(s)+F(1-s)] / 2 \in \Re$. It is clear that $\mathscr{M}$ is convex and, by Theorem 2.2 and the results of Ehrenpreis $[3 ; 4]$, $M \cap \widehat{D}^{v}$ is a neighborhood of zero in $\widehat{D}^{v}$ for each $v$. Thus, $\mathfrak{T}$ is a neighborhood of zero in $\widehat{D}$. Clearly $\mathfrak{T} \cap \mathfrak{T}_{0} D_{m m}=\mathfrak{T}$. We have thus proved

THEOREM 2.3. The topology of $\mathfrak{I}_{0} D_{m m}$ is that induced by the space $\widehat{D}$. In any case, the topology of $\mathfrak{T}_{0} D_{m n}$ is stronger than that induced by $\widehat{D}$. In regard to sequences and bounded sets, the topology of $\mathfrak{T}_{0} D_{m n}$ is that induced by $\widehat{D}$.

The classical theorem of Paley and Wiener was stated for $L_{2}$-functions of compact support rather than indefinitely differentiable functions. In our case the description of the Fourier transform of the space of all $L_{2}$-functions of compact support on $G$, which are of fxed type $m, n$ is an immediate consequence of Theorem 2.1 and Proposi' 0.12 .2 above. Indeed, on the one hand the indefinitely differentiable functions $f(g)$ of type $m, n$ which satisfy $f\left(g_{\zeta}\right)$ $=0$ for $\zeta \geqq \zeta_{0}$ are dense in the space of $L_{2}$-functions of type $m, n$ for which $f\left(g_{\zeta}\right)=0$ for $\zeta>\zeta_{0}$. On the other hand the entire functions $F(s)$ of exponential type $<2 \zeta$ which rapidly decrease on $R s=1 / 2$ are dense in the space $L_{2}(-\infty$ $<t<\infty ; t \tanh \pi t d t)$ of entire functions of exponential type $<2 \zeta$; this denseness is preserved if one restricts oneself to functions $F(s)$ which satisfy the functional equation (2.14) above.

3. The analog of the Paley-Wiener theorem for $D$. In $\S 2$ we characterized the Fourier transform $\mathfrak{T} D_{m n}$ of the space $D_{m n}$ for fixed $m$ and $n$. We now proceed to do this for the whole space $D$. First we need

Lemma 3.1. Let ús choose for each $m, n$ an $f_{m n} \in D_{m n}$. Suppose that for somev (independent of $m$ and $n$ ) we have $f_{m n} \in D_{m n}^{v}$ for all $m$ and $n$. Suppose in addition that, for any polynomial $Q$ in two letters, and any integer $j \geqq 0$ we have

$$
\sup _{\boldsymbol{o}, m, n}\left|Q(m, n)\left(\Delta^{j} f_{m n}\right)(g)\right|<\infty .
$$

Then $\sum f_{m n}$ converges in $D$.

Proof. Let $\vartheta$ denote a suitably normalized element in the Lie algebra of $G$ in the direction of $K$. Then for any $m, n$, we have clearly $\left(L_{\vartheta} f_{m n}\right)(g)=m f_{m n}(g)$, 
$\left(R_{\vartheta} f_{m n}\right)(g)=n f_{m n}(g)$. By making a suitable translation, if necessary, we may assume that we can find a neighborhood of 1 in $G$ such that for no $m, n$ does the support of $f_{m n}(g)$ intersect this neighborhood.

Now, it is clear that the series $\sum f_{m n}$ defines a continuous function $f$ of compact support which vanishes inside of the fixed neighborhood of 1 . In order to show that the series $\sum f_{m n}$ converges in $D$, it is sufficient to consider the operators $\partial / \partial \zeta, L_{\vartheta}$, and $R_{\vartheta}$. Now (see Bargmann [1] formula (4.19)) by subtracting a suitable combination of $L_{\vartheta}$ and $R_{\vartheta}$ from $\Delta$ we obtain an operator $\Delta^{\prime}$ which is a second order differential operator in $\partial / \partial \zeta$ only, whose coefficients are real analytic functions of $\zeta$ for $\zeta>\epsilon$. Now, for the definition of L. Schwartz's topology of the space $D$ on the real line the powers of any second order differential operator with real analytic coefficients may be used in place of the powers of $d / d x$, as is easily verified. It follows that the topology of $D$ (outside the given neighborhood of 1 in $G$ ) can be described by the operators $\Delta^{\prime}, L_{\vartheta}$ and $R_{\vartheta}$. The above implies that for any $p, q, r>0$ the series

$$
\sum \sup _{\boldsymbol{\rho}}\left|\left[\left(\Delta^{\prime}\right)^{p} L_{\vartheta}^{q} R_{\vartheta}^{r} f_{m n}\right](g)\right|
$$

converges. This proves that $\sum f_{m n}$ converges in $D$ which is the desired result.

By similar reasoning we deduce

Lemma 3.2. Let $v>0$; suppose that for each $m, n$ we have a sequence $f_{m n}^{p}$ of functions in $D_{m n}$. Suppose that for each polynomial $Q$ in two letters and each $j$,

$$
\sup _{g, m, n} \mid Q(m, n)\left(\Delta^{j} f_{m n}^{p}\right)(g) \rightarrow 0 .
$$

Then $\sum f_{m n}^{p} \rightarrow 0$ in $D$.

THEOREM 3.1. The following two spaces of matrix-valued functions $F_{m n}(s)$ of one complex variable $s$ are identical:

(a) All $F_{m n}(s)$ of the form $\int_{G} f(g) u_{m n}(g, s) d g$ as $f(g)$ varies over the space $D$ of indefinitely differentiable functions on $G$ of compact support.

(b) All matrix-valued functions $F_{m n}(s)$ which are (i) for fixed $m$ and $n$ contained in $\mathfrak{I}_{0} D_{m n}$; (ii) of exponential type $\leqq v$, where $v$ is independent of $m$ and $n$; (iii) for any polynomial $Q$ in three variables

$$
\begin{aligned}
& \sup _{m, n}\left\{\sup _{R s=1 / 2}\left|Q(m, n, s) F_{m n}(s)\right|\right\}<\infty, \\
& \sup _{m, n}\left\{\sup _{l=1,2, \ldots}\left|Q(m, n, l) f_{m n}^{ \pm}(l)\right|\right\}<\infty
\end{aligned}
$$

where $\left({ }^{4}\right)$, for $0<l \leqq \min (m, n)$ we define $f_{m n}^{+}(l)=\omega_{m n} F_{m n}(-l)$, we define $f_{m n}^{-}(l)$

(4) The $\omega_{m n}(l)$ are defined in (1.10) above. 
$=\omega_{m n} F_{m n}(-l)$ for $0<l<\min (-m,-n)$, and for all other values of $m, n, l$ we define $f_{m n}^{ \pm}(l)=0$.

Thus the algebra $D$ (with convolution as product) is isomorphic to the algebra of all matrix-functions $F_{m n}(s)$ satisfying (b) (with matrix-multiplication as product for each $s$ ).

Proof. By Lemma 2.4, $\sum_{m, n} P_{m n} f$ converges to $f$ in the topology of $D$. Also if $f(g)=f\left(k g_{\zeta} k^{\prime}\right)=0$ for $\zeta>v / 2$, then

$$
\left(P_{m n} f\right)(g)=\iint \chi_{m}(k) \chi_{n}\left(k^{\prime}\right) f\left(k g_{\zeta} k^{\prime}\right) d k d k^{\prime}=0 \quad \text { for } \zeta>v / 2 .
$$

Therefore by Theorem 2.1 and Proposition 2.2, $F_{m n}(s)$ is of exponential type $\leqq v$, noting that

$$
\int f(g) u_{m n}(g, s) d g=\int\left(P_{m n} f\right)(g) u_{m n}(g, s) d g .
$$

Thus $F_{m n}(s) \in \mathfrak{I}_{0} D_{m n}$. This proves that if $f(g) \in D$ then conditions (i) and (ii) of (b) hold for $F_{m n}(s)$. To prove (iii) i.e. (3.1) we observe that for any polynomial $Q_{1}$ of two variables and any $j=0,1,2, \ldots$

$$
\sup _{m, n, \emptyset}\left|Q_{1}(m, n) \Delta^{j}\left(P_{m n} f\right)(g)\right|<\infty
$$

was proved in $\S 2$ above (see Lemma 2.2). Now for $R s=1 / 2$ we have $\left|u_{m n}(g, s)\right| \leqq 1$ and for $l=1,2,3, \cdots$ we have $\left|v_{m n}^{ \pm}(g, l)\right| \leqq 1$, because these are unitary matrices. Moreover the support of $\left(P_{m n} f\right)(g)$ is contained in a compact set independent of $m$ and $n$. Hence for any integer $j$

$$
Q_{1}(m, n) s^{i}(1-s)^{i} F_{m n}(s)=Q_{1}(m, n) \int_{G} \Delta^{j}\left(P_{m n} f\right)(g) u_{m n}(g, s) d g .
$$

Hence by (3.3), $\sup _{m, n, R s=1 / 2}\left|Q_{1}(m, n) s^{j}(1-s)^{i} F_{m n}(s)\right|<\infty$. Moreover for any $j=0,1,2, \cdots$

$$
Q_{1}(m, n) l^{i}(1-l)^{i} f_{m n}^{ \pm}(l)=Q_{1}(m, n) \int \Delta^{j}\left(P_{m n} f\right)(g) v_{m n}^{ \pm}(g, l) d g .
$$

Hence

$$
\sup _{m, n}\left\{\sup _{l=1,2, \ldots, \min (|m|,|n|)}\left|Q_{1}(m, n) l^{i}(1-l)^{i} f_{m n}^{ \pm}(l)\right|\right\}<\infty .
$$

This proves that the class (a) of Theorem 3.1 is contained in the class (b).

Now given $F_{m n}(s)$ satisfying the conditions (b), then for fixed $m, n$ we apply Theorem 2.1 and obtain a unique function $f_{m n}(g) \in D_{m n}$ such that $\mathfrak{T}_{0} f_{m n}=F_{m n}(s)$ for this fixed $m, n$. By (b) condition (ii) all $F_{m n}(s)$ are of exponential type $\leqq v$ where $v$ is independent of $m$ and $n$. Hence by Proposition 
2.2, all $f_{m n}(g)$ have their supports contained in one compact subset of $G$ which is independent of $m$ and $n$. Now set

$$
\begin{aligned}
& f_{m n}^{+}(g)=\sum_{l} \tilde{f}_{m n}^{+}(l)\left(v_{m n}^{+}\right)^{*}(g, l)(2 l-1), \\
& f_{m n}^{-}(g)=\sum_{l} \overline{f_{m n}}(l)\left(v_{m n}^{-}\right)^{*}(g, l)(2 l-1), \\
& f_{m n}^{(0)}(g)=\int F_{m n}(1 / 2+i t)\left(u_{m n}\right)^{*}(g, 1 / 2+i t) \delta t .
\end{aligned}
$$

If $Q_{1}$ is any polynomial in two variables, then

$$
\sup _{m, n, \theta}\left|Q_{1}(m, n) \Delta^{j} f_{m n}^{(0)}(g)\right|<\infty \quad \text { for each } j=0,1,2, \cdots
$$

follows from (3.1) and the fact that

$$
\left|\Delta^{i} u_{m n}(g, s)\right|=\left|s^{j}(1-s)^{i} u_{m n}(g, s)\right| \leqq\left|s^{i}(1-s)^{i}\right| \text { for } R s=1 / 2 \text {. }
$$

Now

$$
f_{m n}^{ \pm}(g)=\sum_{l=1}^{\min (|m|,|n|)}\left(v_{m n}^{ \pm}\right)^{*}(g, l) f_{m n}^{ \pm}(l)(2 l-1)
$$

therefore

$$
\left|f_{m n}^{+}(g)\right| \leqq \sum_{l=1}^{\min (|m|,|n|)}\left|f_{m n}^{ \pm}(l)\right| \cdot(2 l-1) .
$$

Now for any polynomial $Q_{2}$ in two variables we have by (3.1b)

$$
Q_{2}(m, n) f_{m n}^{+}(l)=O\left(m^{-2}\right)
$$

uniformly in $l$. Therefore using (3.10) we conclude

$$
Q_{2}(m, n) f_{m n}^{ \pm}(g)=O(1) \text { uniformly in } g .
$$

Similarly for any $j=0,1,2, \cdots$

$$
Q_{2}(m, n)\left(\Delta^{j} f_{m n}^{ \pm}\right)(g)=O(1) \text { uniformly in } g .
$$

By the Plancherel formula for $G$ (or rather the corresponding Fourier inversion formula) we have

$$
f_{m n}(g)=f_{m n}^{(0)}(g)+\widehat{f_{m n}^{+}}(g)+\widehat{f_{m n}}(g) .
$$

Hence combining (3.7) with (3.11) and Lemma 3.1 we conclude that for any $j=0,1,2, \ldots$ the infinite series $\sum f_{m n}(g)$ converges in the topology of $D$, be- 
cause all $f_{m n}(g)$ have their supports in one compact set. Therefore we obtain a function $f(g) \in D$ such that $f(g)=\sum f_{m n}(g)$ and $\left(P_{m n} f\right)(g)=f_{m n}(g)$. Hence by definition of $\mathfrak{T}$ we have

$$
\mathfrak{I} f=\left\{F_{m n}(s), \hat{f}_{m n}^{+}(l), \overline{f_{m n}}(l)\right\},
$$

hence in particular $\mathfrak{T}_{0} f=\left(F_{m n}(s)\right)$. This completes the proof of Theorem 3.1.

We remark that as an immediate consequence of Theorem 3.1 we obtain the

Corollary. Let $s$ be any fixed complex number, but $s \neq 0, \pm 1, \pm 2, \cdots$. Then the Hilbert space $L_{2}(0 \leqq \theta<1 ; d \theta)$ is irreducible under the operators $U(g, s)$ as $g$ varies over $G$.

On the other hand if $s=0, \pm 1, \pm 2, \cdots$ then there exist proper closed invariant subspaces; as a matter of fact $L_{2}(0 \leqq \theta<1 ; d \theta)$ is not even completely reducible in this case.

In $\S 2$ we described the topology of $\mathfrak{T}_{0} D_{m n}$ for each $m, n$; we shall now derive a similar result for $D$. We denote by $\mathfrak{T}_{0} D$ the space of all matrixvalued functions $F_{m n}(s)$ of the complex variable $s$ which are of the form $\mathfrak{I}_{0} f$, for $f \in D$. From (2.11) and the Fourier inversion formula for $G$, we see that $\mathfrak{T}_{0}$ is one-one. We give $\mathfrak{I}_{0} D$ the topology to make $\mathfrak{I}_{0}$ a topological isomorphism and obtain a topological vector space which we again denote by $\mathfrak{I}_{0} D$. For any $v>0$ we define $D^{v}$ as the space of all $f \in D$ such that $f(g)=0$ whenever $g=k_{\theta} g_{5} k_{\theta^{\prime}}, \zeta \geqq v ; \mathfrak{I}_{0} D^{v}$ is the space of all $\mathfrak{T}_{0} f, f \in D^{v} . D$ is the inductive limit of the spaces $D^{v}$ for $v$ integral. We shall describe first the topology of the spaces $\mathfrak{I}_{0} D^{v}$; since $D^{v}$ is metrizable, it is sufficient to consider sequences.

Theorem 3.2. Let $\left\{F_{m n}^{j}\right\}_{j=1,2, \ldots}$ be a sequence in $\mathfrak{T}_{0} D^{v}$. A necessary and sufficient condition that $F_{m n}^{j} \rightarrow 0$ in $\mathfrak{I}_{0} D^{v}$ is: For any polynomial $Q$ in three variables,

$$
\begin{aligned}
& \sup _{m, n}\left\{\sup _{R s=1 / 2}\left|Q(m, n, s) F_{m n}^{j}(s)\right|\right\} \rightarrow 0, \\
& \sup _{m, n}\left\{\sup _{l=1,2, \ldots}\left|Q(m, n, l) f_{m n}^{ \pm j}(l)\right|\right\} \rightarrow 0
\end{aligned}
$$

where the $f_{m n}^{ \pm j}$ are defined in terms of the $F_{m n}^{i}$ as in Theorem 3.1.

Proof. For each $j$, set $f^{j}=\mathfrak{T}_{0}^{-1} F_{m n}^{j}$. Suppose first that $F_{m n}^{j} \rightarrow 0$ in $\mathfrak{T}_{0} D$, i.e. $f^{i} \rightarrow 0$ in $D$. Then from Lemma 2.3 it follows immediately that, for $Q_{1}$ any polynomial in two variables, and any integer $p \geqq 0$

$$
Q_{1}(m, n) P_{m n} \Delta^{p} f^{i} \rightarrow 0
$$

in $D$ uniformly in $m$ and $n$. (3.12a) now follows by Theorem 2.2. On the other hand, for $l=1,2, \cdots, \min (|m|,|n|),\left|v_{m n}^{ \pm}(g, l)\right| \leqq 1$ because the matrices 
are unitary. Thus, by using the above and

$$
l^{p}(1-l)^{p} f_{m n}^{ \pm j}(l)=\int_{G}\left(\Delta^{p} P_{m n} f^{j}\right)(g) v_{m n}^{ \pm}(g, l) d g
$$

we deduce $(3.12 \mathrm{~b})$ immediately.

Conversely, suppose that the $F_{m n}^{i}$ satisfy (3.12a) and (3.12b). We define $f_{m n}^{+j}(g), f_{m n}^{-j}(g)$, and $f_{m n}^{(0) j},(g)$ as in the proof of Theorem 3.1. Using (3.8) we deduce immediately, for $Q_{1}$ any polynomial in two variables

$$
\sup _{m, n, g}\left|Q_{1}(m, n) \Delta^{p} f_{m n}^{(0) j}(g)\right| \rightarrow 0 \quad \text { for } p=0,1, \cdots .
$$

Using (3.10) and (3.12b) we deduce that, for any polynomial $Q_{2}$ in two variables,

$$
\sup _{m, n, g}\left|Q_{2}(m, n) f_{m n}^{ \pm j}(g)\right| \rightarrow 0
$$

and similarly,

$$
\sup _{m, n, 0}\left|Q_{2}(m, n) \Delta^{p} f_{m n}^{ \pm j}(g)\right| \rightarrow 0 \quad \text { for } p=0,1, \cdots .
$$

On the other hand, by the Fourier inversion formula for $G$, we have

$$
P_{m n} f^{j}=f_{m n}^{(0) j}+f_{m n}^{+j}+f_{m n}^{-i} .
$$

Using (3.13) and (3.14) and the fact that all $f^{j}$ lie in $D^{v}$, we deduce from Lemma 3.2 that $f^{j} \rightarrow 0$ in $D^{v}$, which is the desired result.

For any $v$ we define the space $\widetilde{D}^{v}$ as consisting of all $F_{m n}(s) \in \mathfrak{I}_{0} D$ such that for all $m, n, F_{m n}(s)$ is of exponential type $\leqq 2 v$. The topology of $\widetilde{D}^{v}$ is defined by means of the semi-norms

$$
\sup _{0 \leqq R s \leqq 1, m, n}\left|Q(m, n, s) F_{m n}(s)\right|+\sup _{m, n, l}\left|Q(m, n, l) f_{m n}^{ \pm}(l)\right|
$$

for any polynomial $Q$ in three variables. As an immediate consequence of Theorems 3.1 and 3.2 we have

TheOREm 3.3. $\mathfrak{I}_{0} D$ is the inductive limit of the spaces $\tilde{D}^{v}$ for $v$ integral $>0$.

CoRollary. For any fixed $s_{0}$ the mapping $f \rightarrow\left(F_{m n}\left(s_{0}\right)\right)$ is a continuous mapping of $D$ into the space of all rapidly decreasing matrices $a_{m n}$ of complex numbers with the topology defined by the semi-norms $\sup _{m, n}\left|Q(m, n) a_{m n}\right|$, for any polynomial $Q(m, n)$.

Proof. For $s_{0}$ on the line $R s=1 / 2$ it is an immediate consequence of Theo- 
rem 3.2 that for any $v>0, f \rightarrow\left(F_{m n}\left(s_{0}\right)\right)$ is a continuous map of $D^{v}$ into the space of rapidly decreasing matrices with the above topology. It follows from Theorem 3.3 and the results of Dieudonné and Schwartz [1] that this map is continuous on $D$.

For an arbitrary $s_{0}$, if $f \in D^{v}$, then, by a theorem of Phragmén and Lindelöf (see Titchmarsh [1]), if

$$
\sup _{m, n}\left\{\sup _{R s=1 / 2}\left|Q(m, n) F_{m n}^{i}(s)\right|\right\} \leqq d
$$

for some polynomial $Q$, then also

$$
\sup _{m, n}\left|Q(m, n) F_{m n}^{j}\left(s_{0}\right)\right| \leqq d \exp (|\sigma-1 / 2|)
$$

where $\sigma=R s_{0}$. It follows thus from Theorem 3.2 that $f \rightarrow\left(F_{m n}\left(s_{0}\right)\right)$ is continuous from $D^{v}$ into the space of rapidly decreasing matrices with the above topology. Then, as above, the map is continuous on $D$.

4. Distributions on $G$ and their Fourier transform. Let $D$ be again the space of indefinitely differentiable functions on $G$ with the topology of L. Schwartz as defined in $\$ 2$ above. By $D^{\prime}$ we denote the space of continuous linear complex valued functions defined on $D$. Thus $D^{\prime}$ is the space of distributions in the sense of $\mathrm{L}$. Schwartz on the real analytic manifold $G$. We use on $D^{\prime}$ the topology of uniform convergence on bounded subsets of $D$, the so-called "strong" topology for linear functions. For $f \in D$ and $W \in D$ ' we write

$$
f \cdot W=W \cdot f
$$

for the value of $W$ at $f$. If $w(g)$ is any measurable locally integrable function on $G$ we identify it with the distribution $W$ defined by

$$
W \cdot f=\int_{G} \bar{w}(g) f(g) d g .
$$

We define $P_{m n} W$ for any $W \in D^{\prime}$ by putting

$$
P_{m n} W \cdot f=W \cdot P_{m n} f
$$

and observe that if $W$ is of the form (4.2) then $P_{m n} W \cdot f=\int\left(\left(P_{m n} w\right)\right)^{*}(g) f(g) d g$, where $P_{m n} w$ was defined in $\S 2$ above. Thus in this case the two definitions of $P_{m n}$ agree.

In general, for any function $f$ on $G$ and any $g, x \in G$, define $[L(x) f](g)$ $=f\left(x^{-1} g\right)$ and $[R(x) f](g)=f(g x)$. For any $W \in D^{\prime}, h \in D$, define $L(x) W \cdot h=W$. $L\left(x^{-1}\right) h$ and $R(x) W \cdot h=W \cdot R\left(x^{-1}\right) h$. Then we easily verify

$$
P_{m n} W=\int_{K} \int_{K} \bar{\chi}_{m}(k) \bar{\chi}_{n}\left(k^{\prime}\right) L(k) R\left(k^{\prime}\right) W d k d k^{\prime}
$$


(see (2.1) for the definitions of $\left.\bar{\chi}_{m}, \bar{\chi}_{n}\right)$. For any $f(g)$ we put $\widetilde{f}(g)=f\left(g^{-1}\right)$. For $W \in D^{\prime}, \overleftarrow{W}$ is defined by $\breve{W} \cdot h=\breve{h} \cdot W$ for $h \in D$; and $\bar{W}$ is defined by $\bar{W} \cdot h$ $=(W \cdot \bar{h})^{*}$. For $W \in E^{\prime}, f \in E$ (or $W \in D^{\prime}, f \in D$ ) we define the convolutions $W * f$ and $f * W$ by

$$
(W * f)(g)=\bar{W} \cdot L(g) \widetilde{f} \text { and }(f * W)(g)=\bar{W} \cdot R\left(g^{-1}\right) \widetilde{f} .
$$

We put $D_{m n}^{\prime}=P_{m n} D^{\prime}$ and note that $D_{m n}^{\prime}$ is naturally isomorphic to the dual space of $D_{m n}$, because if $W=P_{m n} W$ then $W \cdot\left(I-P_{m n}\right) f=0$ for all $f \in D$, and thus the restriction of $P_{m n} W$ to $D_{m n}$ determines $P_{m n} W$ uniquely. Moreover the topology of $D_{m n}^{\prime}$ as the dual of $D_{m n}$ is the same as the topology which $D_{m n}^{\prime}$ inherits as a (closed linear) subspace of $D^{\prime}$, because the topology of $D_{m n}$ was defined in $\$ 2$ to be that induced by $D$.

Lemma 4.1. For any $W \in D^{\prime}$ the series $\sum_{m, n} P_{m n} W$ converges to $W$ in the topology of $D^{\prime}$. Moreover, these series converge uniformly for $W$ in any bounded set of $D^{\prime}$.

Proof. Let $B$ be a bounded set in $D$. Then we have shown in Lemma 2.4 that $\sum P_{m n} f$ converges to $f$ in $D$ uniformly for $f \in B$. The result now follows immediately from the definition of $P_{m n} W(4.3)$ and well-known properties of convergence of series in topological vector spaces (see Bourbaki [2]).

The Fourier transform $\mathfrak{T}$ having been defined for $D$ (and $D_{m n}$ ) we can therefore define it for $D^{\prime}$ (and $D_{m n}^{\prime}$ ). Let $f(g) \in D_{m n}$ for fixed $m, n$ then $\mathfrak{T}_{0} f$ is an entire function $F(s)$. Hence if $W \in D_{m n}^{\prime}$ then the equation

$$
(\mathfrak{T} W) \cdot F=W \cdot f
$$

defines a topological isomorphism of $D_{m n}^{\prime}$ onto the dual space $\left(\mathfrak{T}_{0} D_{m n}\right)$ of $\mathfrak{T}_{0} D_{m n}$ because $\mathfrak{T}_{0}$ was a topological isomorphism of $D_{m n}$ onto $\mathfrak{T}_{0} D_{m n}$.

Now let $W$ be an arbitrary element of $D^{\prime}$, then $\mathfrak{T}\left(P_{m n} W\right)$ is defined for each $m$ and $n$, and is an element of the dual of $\mathfrak{I}_{0} D_{m n}$.

Definition 4.1. We call the matrix of distributions $\mathfrak{T}\left(P_{m n} W\right)$ the Fourier transform $\mathfrak{T} W$ of $W$. The $m, n$ coefficient of the matrix $\mathfrak{T}\left(P_{m n} W\right)$ is an element of the dual space of $\mathfrak{I}_{0} D_{m n}$, i.e. a distribution on the topological vector space (of entire functions of exponential type) $\mathfrak{T}_{0} D_{m n}$ explicitly described in $\S 3$. If $\mathfrak{F}(s)=\left[F_{m n}(s)\right] \in \mathfrak{T}_{0} D$ we put

$$
(T W) \cdot \mathfrak{F}(s)=\sum_{m, n}\left(T P_{m n} W\right) \cdot F_{m n}(s) .
$$

We have to prove that this definition is meaningful. Each term in the sum on the right side has meaning because $F_{m n}(s) \in \mathfrak{T}_{0}\left(D_{m n}\right)$ and $\mathfrak{T} P_{m n} W \in\left(T_{0} D_{m n}\right)$. Moreover

$$
\left(\mathfrak{T} P_{m n} W\right) \cdot F_{m n}(s)=P_{m n} W \cdot P_{m n} f=W \cdot P_{m n} f
$$

because $F_{m n}(s)=\mathfrak{I}_{0} P_{m n} f$. Now by Lemma $2.4 \sum_{m, n} P_{m n} f$ converges in the 
topology of $D$ uniformly for $f$ in any bounded set of $D$, hence so does $W\left(\sum_{m, n} P_{m n} f\right)=\sum_{m, n} W \cdot P_{m n} f$. This proves

Lemma 4.2. The right side of (4.5) converges absolutely and uniformly in $f$ and $W$ if $f$ and $W$ remain in bounded subsets of $D$ and $D^{\prime}$ respectively.

We next observe

Lemma 4.3. Let $W \in D^{\prime}$ and $\mathfrak{F} \in \mathfrak{I}_{0} D$. Then

$$
\mathfrak{I} W \cdot \mathfrak{F}=W \cdot f \text { where } \mathfrak{F}=\mathfrak{I}_{0} f .
$$

Proof. If both $W=P_{m n} W$ and $f=P_{m n} f$ then equations (4.6) and (4.4) are the same. If $W$ is an arbitrary element of $D^{\prime}$ and $f$ an arbitrary element of $D$, we know that

$$
\mathfrak{T} W \cdot \mathfrak{F}=\sum_{m, n}\left(T P_{m n} W\right) \cdot F_{m n}(s)
$$

and $W \cdot f=\sum_{m, n} W \cdot P_{m n} f$, where both series converge absolutely and agree termwise. This proves Lemma 4.2.

Now let $w(g) \in L_{2}(G)$, then $\mathfrak{T} w$ was defined previously as a triple $\left\{W_{m n}(s)\right.$, $\left.w_{m n}^{+}(l), w_{m n}^{-}(l)\right\}$. By the Plancherel formula and convention (4.2) we have

$$
\begin{aligned}
W \cdot f= & \int \sum_{m, n} W_{m n}(1 / 2+i t) F_{m n}(1 / 2+i t) \delta t \\
& +\sum_{l} \sum_{m, n}\left[w_{m n}^{+}(l) f_{m n}^{+}(l)+w_{m n}^{-}(l) \overline{f_{m n}}(l)\right](2 l-1) .
\end{aligned}
$$

Thus by Lemma $4.3, \mathfrak{I} W$ is in this case determined by the triple of matrix functions $\left\{W_{m n}(s), w_{m n}^{ \pm}(l)\right\}$ in the sense that the value of $\mathfrak{T} W \cdot \mathfrak{F}$ is the right side of (4.7). But since $f$ is now an element of $D, f_{m n}^{ \pm}(l)$ are determined by $\mathfrak{F}(s)$ (and depend linearly and continuously on $\mathfrak{F}$ ). Hence the right side of (4.7) determines a linear function on $\mathfrak{T}_{0} D$. Thus $\mathfrak{I} W$ and $\mathfrak{T} w$ agree for $w \in L_{2}(G)$.

Now let $\Delta$ be again the two-sided invariant Laplacian on $G$. We know that

$$
\Delta u_{m n}(g, s)=s(1-s) u_{m n}(g, s)
$$

(see Bargmann $[1, \S 6]$ ). If $W \in D^{\prime}$ we define as usual $\Delta W \cdot f=W \cdot \Delta f$.

Lemma 4.4. Let $\mathfrak{F}(s) \in \mathfrak{I}_{0} D . \quad$ Then $\quad \mathfrak{I}_{0} \Delta \mathfrak{T}_{0}^{-1} \mathfrak{F}(s)=s(1-s) \mathfrak{F}(s) . \quad$ Let $W \in\left(\mathfrak{T}_{0} D\right)^{\prime}$, then

$$
\mathfrak{T} \Delta \mathfrak{T}^{-1} W=s(1-s) W .
$$

Proof. For $f \in D$ we have

$$
\mathfrak{T}_{0} f=F_{m n}(s)=\int f(g) u_{m n}(g, s) d g
$$


and

$$
\begin{aligned}
\int(\Delta f)(g) u_{m n}(g, s) & d g=\int f(g)\left(\Delta u_{m n}\right)(g, s) d g \\
= & s(1-s) \int f(g) u_{m n}(g, s) d g=s(1-s) F_{m n}(s)=s(1-s) \mathfrak{T}_{0} f .
\end{aligned}
$$

This proves $\mathfrak{T}_{0} \Delta \mathfrak{T}_{0}^{-1} \mathfrak{F}=s(1-s) \mathfrak{F}$ and the corresponding equation for $W$ follows.

Let us denote by $E$ the space of indefinitely differentiable functions on $G$ with the topology of Schwartz. Then $E$ is a complete, reflexive, metrizable, locally convex topological vector space; a sequence $\left\{f^{j}\right\} \rightarrow 0$ in $E$ if and only if the functions $f^{i}$ and all their derivatives $\rightarrow 0$ uniformly on every compact set of $G$. By $E^{\prime}$ we denote the dual of $E$ with the topology of uniform convergence on the bounded sets of $E$; it is known that $E^{\prime}$ consists of all distributions on $G$ of compact support.

For any $f \in E, P_{m n} f$ is defined by (2.1); for any $W \in E^{\prime}, P_{m n} W$ is defined by (4.3). (Note that, since $D$ is dense in $E$, any $W \in E^{\prime}$ is uniquely determined by its restriction to $D$.) It is clear that $P_{m n} W \in E^{\prime}$. Moreover we have

LemMa 4.5. The series $\sum P_{m n} f$ converges to $f$ in $E$ uniformly for $f$ in any bounded subset of $E$. Similarly, the series $\sum P_{m n} W$ converges to $W$ in $E^{\prime}$ uniformly for $W$ in any bounded subset of $E^{\prime}$.

Proof. The proof of the first part of Lemma 4.5 is exactly the same as the proof of Lemma 2.4. The second part then follows as in the proof of Lemma 4.1.

Corollary. For any $W, X \in E^{\prime}$ we have $P_{m n}(W * X)=\sum_{j} P_{m j} W * P_{j n} X$ where the series converges in $E^{\prime}$.

Proof. It follows immediately from the orthonormality of the characters of $K$ that, if $j \neq i, P_{m j} W * P_{i n} X=0$. The result now follows immediately from the continuity of convolution and known results on sums of vector-valued maps (see Bourbaki [2]).

Denote by $E_{m n}^{\prime}$ the space of all $W \in E^{\prime}$ which satisfy $P_{m n} W=W . E_{m n}^{\prime}$ is given the topology induced by $E^{\prime}$. Then by the results of Ehrenpreis. [2] (see also Schwartz [4]) we have

PROPOSITION 4.1. $E_{m n}^{\prime}$ consists of all $W \in D_{m n}^{\prime}$ such that the convolution $W * f \in D_{m n}$ for all $f \in D_{n n}$. In regards to bounded sets and sequential convergence, the topology of $E_{m n}^{\prime}$ is the same as the compact-open topology of the space of linear transformations $f \rightarrow W * f$ of $D_{n n}$ into $D_{m n}$.

For any $W \in E^{\prime}$, we can find a finite number of polynomials $\partial_{1}, \partial_{2}, \cdots, \partial_{r}$ in elements of the Lie algebra of $G$ (considered as acting on functions on $G$ by 
left differentiation) and a corresponding sequence of measures of compact support $\mu_{1}, \mu_{2}, \cdots, \mu_{r}$ on $G$ so that

$$
W \cdot f=\sum_{j=1}^{r} \int \partial_{j} f d \mu_{j}
$$

We shall now proceed to characterize the Fourier transform on $E^{\prime}$. As in the case of the space $D$, we study first the Fourier transform on $E_{m n}^{\prime}$. The case $m=n=0$ was treated by Ehrenpreis and Mautner [1].

ThEOREM 4.1. Let $m$ and $n$ be fixed integers. The following classes of functions of the complex variable s are identical:

(a) All functions of $s$ of the form $W \cdot u_{m n}(g, s)$ for $W \in E_{m n}^{\prime}$.

(b) All entire functions $W(s)$ of exponential type which are slowly increasing on the line $R s=1 / 2$ and which satisfy (2.14) and

$$
W(1-s)=\chi_{m n}(s) W(s) .
$$

The function $H(1 / 2+i t)$ is said to be slowly increasing if $H(1 / 2+i t)$ $=\phi(1+|t| r)$ for some $r$. Note that any function $W$ which satisfies (4.10) also satisfies

$$
\begin{array}{r}
W(s)=0 \text { for } s=-|m|,-|m|-1, \cdots,-|n|+1 \text { if }|n|>|m| \\
\quad \text { or for } s=-|n|,-|n|-1, \cdots,-|m|+1 \text { if }|m|>|n| .
\end{array}
$$

Proof. Let $\Delta$ be again the left and right invariant Laplacian on $G$ and $\xi_{1}, \xi_{2}$ as in $\$ 2$ above (see formulae (2.16), (2.17) and (2.18)). By forming polynomials in $\Delta, \xi_{1}, \xi_{2}$ we can obtain $\vartheta^{2}$ where $\vartheta$ is in the Lie algebra of $K$ and $\vartheta \neq 0$ (see for example Bargmann [1, (4.17), (4.18) on p. 596 and (6.22) on p. 613]). But convergence of each even derivative $\partial^{2 n} / \theta^{2 n}$ implies convergence of each derivative $\partial^{n} / \partial \theta^{n}$. Hence the topology of $E$ is determined by $\vartheta^{2}, \xi_{1}, \xi_{2}$ since $\vartheta, \xi_{1}$ and $\xi_{2}$ generate the associative enveloping algebra of the Lie algebra of $G$. Therefore the topology of $E$ is also determined by $\Delta, \xi_{1}$ and $\xi_{2}$ in the sense that $F$ is sufficient to verify convergence in $E$ by applying every (noncommutative) polynomial in $\Delta, \xi_{1}$ and $\xi_{2}$ to the elements of $E$.

Making use of (4.10), Theorem 1 of Part I, and the fact that $\left|u_{i j}(g, s)\right|$ $\leqq u_{00}(g, 0)$ for any $i, j$ it follows that, for any $W \in E_{m n}^{\prime}$, the function $W(s)$ defined by $s \rightarrow W \cdot u_{m n}(g, s)$ is an entire function of exponential type which is slowly increasing on $R s=1 / 2$. The fact that (4.11) and (2.14) are satisfied for $W(s)$ follows from the fact that they are satisfied by $u_{m n}(g, s)$ and hence for all its derivatives with respect to $g$. This proves that the class (a) is contained in the class (b).

We now define, for any $W(s)$ of the class (b),

$$
\begin{aligned}
& w^{+}(l)=\omega_{m n}(l) W(-l) \text { for } l=1,2, \cdots, \min (m, n), \\
& w^{-}(l)=\omega_{m n}(l) W(-l) \text { for } l=1,2, \cdots, \min (-m,-n) .
\end{aligned}
$$


Then we claim that the triple $\left\{W(s), w^{+}(l), w^{-}(l)\right\}$ defines $\mathfrak{T} W$ in the sense that, for any $f \in D_{m n}$, if $\mathfrak{T} f=\left\{F(s), f^{+}(l), f^{-}(l)\right\}$ then

$$
\begin{aligned}
W \cdot f= & \int_{R s=1 / 2} W(s) F(s) \delta s \\
& +\sum_{l} w^{+}(l) f^{+}(l)(2 l-1)+\sum_{l} w(l) f^{-}(l)(2 l-1) .
\end{aligned}
$$

By (4.10), and the Fourier inversion formula for $G$, we may write

$$
\begin{aligned}
W \cdot f= & \sum_{j=1}^{r} \int\left(\partial_{j} f\right)(g) d \mu_{j}(g) \\
= & \sum_{j=1}^{r} \int d \mu_{j}(g) \partial_{j} \int_{R,=1 / 2} F(s)\left(u_{m n}\right)^{*}(g, s) \delta s \\
& +\sum_{j=1}^{r} \int d \mu_{j}(g) \partial_{j} \sum_{l} f^{+}(l)\left(v^{+}\right)^{*}(g, l)(2 l-1) \\
& +\sum_{j=1}^{r} \int d \mu_{j}(g) \partial_{j} \sum_{l} f^{-}(l)\left(v^{-}\right)^{*}(g, l)(2 l-1) .
\end{aligned}
$$

Since $F \in \mathfrak{T}_{0} D_{m n}$, it is not difficult to show that $\partial_{j}$ can be applied under the integral sign in the right side of (4.14). Since $\mu_{j}$ are measures of compact support on $G$, we may invert the order of integration in (4.15) to obtain

$$
\begin{aligned}
W \cdot f= & \int_{R_{s=1 / 2}} F(s) \delta s \int \sum_{j} \partial_{j}\left(u_{m n}\right)^{*}(g, s) d \mu_{j}(g) \\
& +\sum_{l} f^{+}(l)(2 l-1) \int \sum_{j} \partial_{j}\left(v^{+}\right)^{*}(g, l) d \mu_{j}(g) \\
& +\sum_{l} f^{-}(l)(2 l-1) \int \sum_{j} \partial_{j}\left(v^{-}\right)^{*}(g, l) d \mu_{j}(g) \\
= & \int_{R_{s=1 / 2}} W(s) F(s) \delta s+\sum_{l} w^{+}(l) f^{+}(l)(2 l-1)+\sum_{l} w^{-}(l) f^{-}(l)(2 l-1) .
\end{aligned}
$$

This proves (4.14).

Let us observe that, if $W$ is of the form (4.2) with $w \in D_{m n}$ then for any $h \in D_{n n}$ we have

$$
\int(W * h)(g) u_{m n}(g, s) d g=\left[\mathfrak{T}_{0}(w * h)\right](s)=\left[\left(\mathfrak{T}_{0} w\right)(s)\right]\left[\left(\mathfrak{T}_{0} h\right)(s)\right] .
$$

By continuity, and the fact that distributions of the form (4.2) are dense in $E^{\prime}$, we deduce that, for any $W \in E^{\prime}$, 


$$
\int(W * h)(g) u_{m n}(g, s) d g=[W(s)]\left[\left(\mathfrak{T}_{0} h\right)(s)\right]
$$

By similar reasoning on the discrete series we have

$$
\mathfrak{T}(W * h)=\left\{W(s) H(s), w^{+}(l) h^{+}(l), w^{\left.-(l) h^{-}(l)\right\}}\right.
$$

where

$$
\mathfrak{T} h=\left\{H(s), h^{+}(l), h^{-}(l)\right\} .
$$

Then it follows immediately from Theorem 2.1 that, for any $h \in D_{n n}$ if

$$
\mathfrak{T} h=\left\{H(s), h^{+}(l), h^{-}(l)\right\},
$$

then

$$
\left\{W(s) H(s), w^{+}(l) h^{+}(l), w^{-}(l) h^{-}(l)\right\} \in \mathfrak{T} D_{m n} .
$$

Now, it follows easily from Theorem 2.2 that the map

$$
\begin{aligned}
& \left\{F(s), f^{+}(l), f^{-}(l)\right\} \\
& \quad \rightarrow \int_{R_{s=1 / 2}} F(s) W(s) \delta s+\sum f^{+}(l) w^{+}(l)(2 l-1)+\sum f^{-}(l) w^{-}(l)(2 l-1)
\end{aligned}
$$

defines a continuous linear function $X$ on $\mathfrak{T} D_{m n}$. Thus, there is a $W \in D_{m n}^{\prime}$ with $\mathfrak{T} W=X$.

For any $h \in D_{n n}, f \in D_{m n}$, we have

$$
W * h \cdot f=W \cdot f * h .
$$

If we write $\mathfrak{T} h=\left\{H(s), h^{+}(l), h^{-}(l)\right\}$ and $\mathfrak{T} f=\left\{F(s), f^{+}(l), f^{-}(l)\right\}$ then the above yields

$$
\begin{aligned}
W \cdot f * h= & \mathfrak{T} w \cdot \mathfrak{T}(f * h) \\
= & \int W(s) F(s) H(s) \delta s+\sum w^{+}(l) f^{+}(l) h^{+}(l)(2 l-1) \\
& +\sum w^{-}(l) f^{-}(l) h^{-}(l)(2 l-1) .
\end{aligned}
$$

This shows that

$$
\mathfrak{T}(W * h)=\left\{W(s) H(s), w^{+}(l) h^{+}(l), w^{-}(l) h^{-}(l)\right\} .
$$

We have already remarked that $\left\{W(s) H(s), w^{+}(l) h^{+}(l), w^{-}(l) h^{-}(l)\right\}$

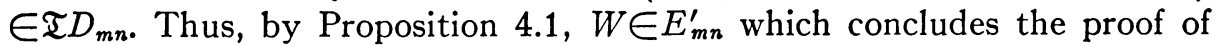
Theorem 4.1.

Let us remark that, in the course of the proof of Theorem 4.1 we have obtained

Proposition 4.2. For any $W \in E_{m n}^{\prime}$ the triple $\left\{W(s), w^{+}(l), w^{-}(l)\right\}$ as de- 
fined in the proof of Theorem 4.1 represents $W$ in the sense that (4.14) holds.

Corollary. Any $W \in E_{m n}^{\prime}$ is determined by $W_{m n}(s)=W \cdot u_{m n}(g, s)$.

We shall, in the future, write $\mathfrak{T} W=\left\{W(s), w^{+}(l), w^{-}(l)\right\}$, and $\mathfrak{I}_{0} W$ $=W(s)$, with $\left\{W(s), w^{+}(l), w^{-}(l)\right\}$ defined in terms of $W$ as in the proof of Theorem 4.1.

The proof of the following proposition is contained in the proof of Theorem 4.1:

Proposition 4.3. For any $W \in E_{r m}^{\prime}, h \in D_{m n}$, if $\mathfrak{T}_{0} W=W(s)$ and $\mathfrak{I}_{0} h=H(s)$ then $\mathfrak{I}_{0}(W * h)=W(s) H(s)$.

Proposition 4.4. For any $W \in E_{r m}^{\prime}, X \in E_{m n}^{\prime}$ if $\mathfrak{I}_{0} W=W(s), \mathfrak{I}_{0} X=X(s)$ then $\mathfrak{I}_{0}(W * X)=W(s) X(s)$.

Proof. For any $h \in D_{n n}$, if $\mathfrak{T}_{0} h=H(s)$ then, by Proposition 4.3, $\mathfrak{T}_{0}(X * h)$ $=X(s) H(s)$. Thus, again by Proposition 4.3, $\mathfrak{I}_{0}(W * X * h)=W(s) X(s) H(s)$. The result now follows since $X=\lim _{E}, X * h$ for appropriate $h \in D_{n n}$.

For any $W \in E^{\prime}$, let us denote by $\mathfrak{I} W$ the triple of matrices

$$
\mathfrak{T} W=\left\{W_{m n}(s), w_{m n}^{+}(l), w_{m n}^{-}(l)\right\}
$$

where

$$
\mathfrak{T} W_{i j}(W)=\left\{W_{i j}(s), w_{i j}^{+}(l), \widetilde{w_{i j}^{-}}(l)\right\}
$$

for any $i, j$. We write

$$
\mathfrak{I}_{0} W=\left\{W_{m n}(s)\right\} .
$$

Proposition 4.5. Let $W, X \in E^{\prime}, \mathfrak{I}_{0} W=\left\{W_{m n}(s)\right\}, \mathfrak{I}_{0} X=\left\{X_{m n}(s)\right\}$. Then

$$
\mathfrak{I}_{0}(W * X)=\left\{\sum_{j} W_{m j}(s) X_{j n}(s)\right\}
$$

where the series on the right side of (4.19) converges uniformly for s in any compact set of the complex plane.

Proof. It follows from Lemma 1.2 that, for fixed $m, n$, the functions of $g: u_{m n}(g, s)$ form a bounded set in $E$ whenever $s$ stays in a fixed compact set of the complex plane. Thus, the maps

$$
Y \rightarrow Y \cdot u_{m n}(g, s)
$$

are equicontinuous linear functions on $E^{\prime}$ for $s$ in any compact set. The result now follows immediately from Proposition 4.4 and the corollary to Lemma 4.3 .

PROPOSITION 4.6. Let $W \in E_{m n}^{\prime}$ have the property that 
(4.20) $W \cdot f=0$ whenever $f \in D$ and $f\left(k g_{5} k^{\prime}\right)=0$ for $k, k^{\prime} \in K, \zeta \geqq v$.

Then the exponential type of $\mathfrak{I}_{0} W$ is $\leqq 2 v$. Conversely, if $\mathfrak{I}_{0} W$ is of exponential type $\leqq 2 v$ then $W$ satisfies (4.20).

Proof. We know that $W=\lim _{E_{m n}^{\prime}} W * h^{j}$ where $h^{j}$ is a sequence in $D_{n n}$ with the supports of $h^{j}\left(g_{\zeta}\right)$ as small as we please, i.e. $h^{j}\left(g_{\zeta}\right)=0$ for $\zeta>\epsilon$. The result now follows immediately from Propositions 2.2 and 4.3.

We can now describe explicitly the Fourier transform $\mathfrak{I}_{0}$ of $E^{\prime}$ :

THEOREM 4.2. The following classes of matrices of entire functions of the complex variable s are identical:

(a) $A l l\left\{F_{m n}(s)\right\}=\left\{\mathfrak{I}_{0} P_{m n} W\right\}$ as $W$ varies over $E^{\prime}$.

(b) All matrices $\left\{F_{m n}(s)\right\}$ satisfying for each $m, n$ the functional equation (2.13) and conditions (2.14) and

(1) The $F_{m n}(s)$ are of fixed exponential type independent of $m$ and $n$.

(2) For any positive integer $r$ we can find positive integers $b$ and $j$ such that

$$
\begin{aligned}
F_{m n}\left(\frac{1}{2}+i t\right) & =O\left[\frac{\left(1+|t|^{b}\right)\left(1+|m|^{j}\right)}{1+|n|^{r}}\right], \\
F_{m n}\left(\frac{1}{2}+i t\right) & =O\left[\frac{\left(1+|t|^{b}\right)\left(1+|n|^{j}\right)}{1+|m|^{r}}\right], \\
f_{m n}^{ \pm}(l) & =O\left[\frac{\left(1+3 l^{b}\right)\left(1+|m|^{j}\right)}{1+|n|^{r}}\right], \\
f_{m n}^{ \pm}(l) & =O\left[\frac{\left(1+l^{b}\right)\left(1+|n|^{j}\right)}{1+|m|^{r}}\right]
\end{aligned}
$$

where $f_{m n}^{ \pm}(l)=\omega_{m n}(l) F_{m n}(-l)$ for any integer $l \geqq 1$.

REMARK. It is not difficult to see that the matrices of class (b) of Theorem 4.2 form an algebra under matrix multiplication. This algebra is isomorphic to the algebra $E^{\prime}$ (under convolution) by Proposition 4.4.

Proof. It follows from Lemmas 1.2 and 1.3 that, given any left differential operator $\partial$ which is a polynomial in the elements of the Lie algebra of $G$, and given any compact set $C$ in $G$ and given any positive integer $r$, we can find positive integers $b$ and $j$ such that

$$
\begin{aligned}
& \partial u_{m n}\left(g, \frac{1}{2}+i t\right)=O\left[\frac{\left(1+|t|^{b}\right)\left(1+|m|^{j}\right)}{1+|n|^{r}}\right], \\
& \partial u_{m n}\left(g, \frac{1}{2}+i t\right)=O\left[\frac{\left(1+|t|^{b}\right)\left(1+|n|^{j}\right)}{1+|n|^{r}}\right],
\end{aligned}
$$




$$
\begin{aligned}
& \partial v_{m n}^{ \pm}(g, l)=O\left[\frac{\left(1+l^{b}\right)\left(1+|m|^{j}\right)}{1+|n|^{r}}\right], \\
& \partial v_{m n}^{ \pm}(g, l)=O\left[\frac{\left(1+l^{b}\right)\left(1+|n|^{j}\right)}{1+|m|^{r}}\right]
\end{aligned}
$$

where the symbol $O$ may be taken uniformly for $g \in C$. We use the fact that for any $r \geqq 0$ we can find a constant $c$ such that for all complex numbers $z, z^{\prime}$ we have $\left(1+\left|z-z^{\prime}\right| r\right)^{-1} \leqq c(1+|z| r)\left(1+\left|z^{\prime}\right| r\right)^{-1}$. From Proposition 4.5 and Equation 4.10 it follows thus that the class (a) is contained in the class (b).

To prove the converse, let $\left\{F_{m n}(s)\right\}$ satisfy condition (b). Then, it follows immediately from Theorem 3.2 that the map

$$
\begin{aligned}
\left\{A_{m n}(s), a_{m n}^{+}(l),\right. & \left.a_{m n}^{-}(l)\right\} \rightarrow \sum_{m, n} \int F_{m n}(s) A_{m n}(s) \delta s \\
& +\sum_{m, n, l} \overline{f_{m n}^{+}}(l) a_{m n}^{+}(l)(2 l-1)+\sum_{m, n, l} \overline{f_{m n}}(l) \overline{a_{m n}^{-}}(l)(2 l-1)
\end{aligned}
$$

defines a continuous linear function $X$ on $\mathfrak{T} D$. Thus, owing to the fact that $\mathfrak{T}$ is a topological isomorphism, we can find a $W \in D^{\prime}$ such that $\mathfrak{T} W=X$.

Now, for any $a, b \in D$ if $\mathfrak{T} a=\left\{A_{m n}(s), a_{m n}^{+}(l), a_{m n}^{-}(l)\right\}$, then

$$
\begin{aligned}
& W * a \cdot b=W \cdot a * b \\
& =\mathfrak{I} W \cdot \mathfrak{T}(a * b) \\
& =\mathfrak{T} W \cdot \int \sum_{j} A_{m j}(s)\left(B_{n j}\right)^{*}(s), \sum_{j} \bar{a}_{m j}^{+}(l)\left(b_{n j}^{+}\right)^{*}(l), \sum_{j} \overline{a_{m j}}(l)\left(\overline{b_{n j}}\right)^{*}(l) \\
& =\sum_{j, m, n} \int_{R_{s=1 / 2}} F_{m n}(s) A_{m j}(s) B_{n j}(s) \delta s \\
& +\sum_{j, m, n, l} \overline{f_{m n}^{+}(l)} a_{m n}^{+}(l)\left(b_{n j}^{+}\right) *(l)(2 l-1)+\sum_{i, m, n, l} \overline{f_{m n}}(l) \overline{a_{m j}}(l)\left(\overline{b_{n j}}\right)^{*}(l)
\end{aligned}
$$

where, because of our hypotheses and Theorem 3.1, it follows easily that we have absolute convergence on the right side of (4.25).

It is an immediate consequence of our hypotheses and Theorem 3.1 that the triple

$$
\left\{\sum_{n} F_{m n}(s) B_{n j}(s), \sum_{n} \bar{f}_{m n}^{+}(l) b_{n j}^{+}(l), \sum_{n} \overline{f_{m n}}(l) \overline{b_{n j}}(l)\right\}
$$

is an element of $\mathfrak{T} D$. By (4.25) this triple is nothing but $\mathfrak{T}(W * b)$. The result now follows immediately from the following proposition (see Ehrenpreis [2]), the second part of which will be used later:

Proposition 4.7. Let $W \in D^{\prime}$; then $W \in E^{\prime}$ if and only if $W * a \in D$ for every $a \in D$. Consider each $W \in E^{\prime}$ as defining the continuous linear map 
$a \rightarrow W * a$ of $D \rightarrow D$, and give this set of maps the compact-open topology J. (That is, a fundamental system of neighborhoods of zero for $J$ consists of those sets $N \subset E^{\prime}$ for which we can find a set $\bar{L}$ in $D$ and a neighborhood of zero $M$ in $D$ so that $N$ consists of those $W \in E^{\prime}$ for which $W * a \in M$ whenever $a \in \bar{L}$.) Then the topology of $E^{\prime}$ is the same as 3 .

We have already shown (see Theorem 2.3 ) that for each $m, n$, the topology of $\mathfrak{I}_{0} D_{m n}$ is, regarding sequences and bounded sets, that induced by the space $\widehat{D}$ of L. Schwartz. Now, define $\mathfrak{I}_{0} E_{m n}^{\prime}$ as the space of all $\mathfrak{I} W$ for $W \in E_{m n}^{\prime}$; we give $\mathfrak{T}_{0} E_{m n}^{\prime}$ the topology to make the Fourier transform a topological isomorphism. Denote by $\widehat{\mathcal{E}}^{\prime}$ the ordinary Fourier transform of the usual space of distributions of compact support on the real line (after a change of variable $s \rightarrow 1 / 2+i s$; see $(0.2)$ and $(0.3))$.

Proposition 4.8. A set $B \subset \mathfrak{I}_{0} E_{m n}^{\prime}$ is bounded if and only if the following conditions hold:

(a) All $F(s) \in B$ are of exponential type $\leqq r$, where $r$ depends only on $B$.

(b) There exist positive numbers $M, \alpha$ so that, for all $F(s) \in \circledast$,

$$
\sup \frac{|F(1 / 2+i t)|}{1+|t|^{\alpha}} \leqq M .
$$

Proof. Suppose first that $B$ satisfies conditions (a) and (b). Then, if $C$ is any bounded set in $\mathfrak{I}_{0} D_{n n}$ it follows immediately from Theorem 2.2 that $\{F(s) H(s)\}_{F(s) \in B, H,(s) \in C}$ is bounded in $\mathfrak{I}_{0} D_{m n}$. From Propositions 4.1 and 4.3 we deduce that $B$ is bounded in $\mathfrak{T}_{0} E_{m n}^{\prime}$.

Conversely, suppose $B$ is bounded in $\mathfrak{I}_{0} E_{m n}^{\prime}$ and denote by $B^{*}$ the set $\left\{\mathfrak{T}_{0}^{-1} F(s)\right\}_{F \in \Theta}$, so that $B^{*}$ is bounded in $E_{m n}^{\prime}$. It follows from well-known results that all $W \in B^{*}$ have their supports in a fixed compact set of $G$. Thus, by Proposition 4.6, all $F(s) \in \Theta$ are of exponential type $\leqq r$, where we may choose $r$ depending on $B$ only.

It is also known that there exists a neighborhood of zero $N$ in $E_{m n}$ so that, for all $W \in B^{*}, f \in N$, we have $|W \cdot f| \leqq 1$. Now, it follows immediately from Lemma 1.2 and the definition of the topology of $E_{m n}$ that we can find positive numbers $\alpha, M$ so that, for $t$ real, we have

$$
\frac{u_{m n}(g, 1 / 2+i t)}{M\left(1+|t|^{\alpha}\right)} \in N .
$$

The result is now immediate.

Proposition 4.9. The topology of $\mathfrak{I}_{0} E_{m n}^{\prime}$ is stronger than that induced by the space $\hat{\mathcal{E}}^{\prime}$ of Schwartz.

Proof. It has been shown recently by A. Grothendieck [1] that the space $\mathcal{E}^{\prime}$ is bornologic; a similar proof shows that $E_{m n}^{\prime}$ is bornologic. (A locally convex topological vector space $\mathfrak{H C}$ is said to be bornologic if any set $N \subset Z$ which 
has the property that, for any bounded set $B \subset Z$ we can find an $a>0$ so that $a ß \subset N$, is a neighborhood of zero in $Z$.) Now, denote by $J$ the topology induced by $\hat{\mathcal{E}}^{\prime}$ on the set of functions of $\mathfrak{T}_{0} E_{m n}^{\prime}$. Thus, the bounded sets of $\mathfrak{J}$ are the same as those of $\mathfrak{T}_{0} E_{m n}^{\prime}$ by Proposition 4.8. Since, among all topologies which can be defined on the set of functions of $\mathfrak{I}_{0} E_{m n}^{\prime}$ which have the same bounded sets the bornologic space has the strongest topology, the result follows.

Proposition 4.10. A sequence $\left\{F^{j}(s)\right\} \subset \mathfrak{T}_{0} E_{m n}^{\prime}$ converges to zero in $\mathfrak{T}_{0} E_{m n}^{\prime}$ if and only if $\leqq r$.

(a) There exists an $r$ independent of $j$ so that all $F^{j}$ are of exponential type

(b) There exists an $\alpha>0$ so that

$$
\sup _{-\infty<i<\infty} \frac{\left|F^{i}(1 / 2+i t)\right|}{1+|t|^{\alpha}} \rightarrow 0 .
$$

Proof. Suppose first that $\left\{F^{i}\right\}$ satisfies (a) and (b). Then for any bounded set $\triangle \subset \mathfrak{T}_{0} D_{n n}$ it follows from Theorem 2.2 that $F^{j}(s) H(s) \rightarrow 0$ in $\mathfrak{T}_{0} D_{m n}$ uniformly for $H \in B$. It results from Propositions 4.1 and 4.3 that $F^{j} \rightarrow 0$ in $\mathfrak{T}_{0} E_{m n}^{\prime}$.

On the other hand, suppose $\left\{F^{j}(s)\right\} \rightarrow 0$ in $\mathfrak{T}_{0} E_{m n}^{\prime}$, and write, for each $j$, $W^{i}=\mathfrak{I}_{0}^{-1} F^{j}(s)$. Then it is known (see Schwartz [4, vol. I, p. 91] where the analogous result for the real line is proved) that there exists a neighborhood $N$ of zero in $E_{m n}$ so that $W^{i} \cdot f \rightarrow 0$ uniformly for $f \in N$. By Theorem 1 we can find positive numbers $M, \alpha$ so that (4.27) holds. Proposition 4.10 results immediately.

Putting together Propositions 4.7, 4.8 and 4.10 with the explicit expression of the topology of $\widehat{\mathcal{E}}^{\prime}$ we have

THEOREM 4.3. In regard to bounded sets and sequential convergence, the topology of $\mathfrak{I}_{0} E_{m n}^{\prime}$ is that induced by $\widehat{\mathcal{E}}^{\prime}$.

Denote by $\mathfrak{T} E^{\prime}$ the space of all $\mathfrak{I} W$ for $W \in E^{\prime}$; the topology of $\mathfrak{T} E^{\prime}$ is defined to make $\mathfrak{T}$ a topological isomorphism.

By reasoning similar to the proofs of Propositions 4.8 and 4.10 we have

TheOREM 4.4. Let $@$ be a set of triples $\left\{F_{m n}(s), f_{m n}^{+}(l), f_{m n}^{+}(l)\right\}$ in $\mathfrak{T} E^{\prime} . \otimes$ is bounded in $\mathfrak{T} E^{\prime}$ if and only if

(a) We can find a number $e$ depending only on $B$ so that all $F_{m n}(s)$ are of exponential type $\leqq$.

(b) For any integer $r$ we can find integers $i$ and $j$ so that (4.21a) and (4.21b) hold where the symbols $\varnothing$ in these formulae may be chosen independent of $\left\{F_{m n}(s), f_{m n}^{+}(l), f_{m n}^{-}(l)\right\} \in B$.

Let $\left\{\left\{F_{m n}^{j}(s), f_{m n}^{+f}(l), f_{m n}^{-j}(l)\right\}\right\}$ be a sequence of triples in $\mathfrak{T} E^{\prime}$. Then this sequence converges to zero in $E^{\prime}$ if and only if 
(a) We can find an $e$ independent of $j$ so that all $P_{m n}^{i}(s)$ are of exponential type $\leqq e$. that

(b) Given any positive integer $r$ we can find positive integers $i$ and $q$ so

$$
\begin{aligned}
\sup _{t, m, n} \frac{\left(1+|n|^{r}\right)\left|F_{m n}^{j}(1 / 2+i t)\right|}{\left(1+|t|^{i}\right)\left(1+|m|^{q}\right)} & \rightarrow 0, \\
\sup _{l, m, n} \frac{\left(1+|m|^{r}\right)\left|F_{m n}^{j}(1 / 2+i t)\right|}{\left(1+|t|^{i}\right)\left(1+|n|^{q}\right)} & \rightarrow 0, \\
\sup _{l, m, n} \frac{\left(1+|n|^{r}\right)\left|f_{m n}^{ \pm j}(l)\right|}{\left(1+l^{i}\right)\left(1+|m|^{q}\right)} & \rightarrow 0, \\
\sup _{l, m, n} \frac{\left(1+|m|^{r}\right)\left|f_{m n}^{ \pm j}(l)\right|}{\left(1+l^{i}\right)\left(1+|n|^{q}\right)} & \rightarrow 0 .
\end{aligned}
$$

We shall now use the above theory to deduce some important results about the algebra $E^{\prime}$.

Proposition 4.11. There exists no $W \in E^{\prime}, W \neq 0$ such that, for each $m, n$,

$$
W \cdot u_{m n}(g, s)=0 \text { for a nondiscrete set of } s \text {. }
$$

Proof. For each $m, n, \mathfrak{T}_{0} P_{m n} W$ is an entire function of exponential type by Theorem 4.1. Thus, if (4.30) were satisfied, we would have $\mathfrak{I}_{0} P_{m n} W=0$ for all $m$ and $n$. By the corollary to Proposition 4.2 it would then follow that $P_{m n} W=0$. Since $W=\sum P_{m n} W$, by Lemma 4.5 , we would have $W=0$.

Proposition 4.12. The closure in $L_{2}(G)$ of the linear combinations of the matrix coefficients of the discrete series of irreducible unitary representations does not contain any function on $G$ of compact support.

Proof. Suppose $f(g)$ is of compact support and $f(g)=\lim _{L_{\mathbf{g}}(G)} h(g)$ where $h(g)$ are linear combinations of the matrix coefficients of the discrete series. (Bargmann [1] has shown that these matrix coefficients are in $L_{2}(G)$.) Then for any real $t$, and any $m, n$

$$
\int(h(g))^{*} u_{m n}(g, 1 / 2+i t) d g=0
$$

because of the orthogonality relations for the unitary representations of $G$ (see Bargmann [1]). Thus, by the Plancherel formula on $G$, we have also 


$$
\int(f(g))^{*} u_{m n}(g, 1 / 2+i t) d g=0
$$

for all $m, n, t$. We now apply to the distribution $f(g)$ (see (4.2)) Proposition 4.11 and obtain the desired result.

From Proposition 4.11 we deduce by duality

PROPOSITION 4.13. The linear combinations of the $u_{m n}(g, 1 / 2+i t)$ for varying $m, n$ and $t$ are dense in $E$. In particular, if $v(g)$ is a coefficient of a matrix in the discrete series then $v(g)$ is the limit of linear combinations of the $u_{m n}(g$, $1 / 2+i t)$.

REMARK. Proposition 4.13 is remarkable because we know, by the orthogonality relations for unitary representations of $G$, that $v(g)$ is "orthogonal" to all $u_{m n}(g, 1 / 2+i t)$ in the sense of the $L_{2}$-theory for $G$.

An important principle of Harish-Chandra in his work on the theory of representations of semi-simple Lie groups is that there are "sufficiently many" finite dimensional representations to determine the algebraic relations in the associative enveloping algebra of the Lie algebra. For our group $G$ the finite-dimensional representations may be described as follows: For each positive integer $l$ we have a representation $\pi(g, l)$ of degree $2 l+1$. The matrix coefficients of $\pi(g, l)$ may be written as (see for example Bargmann [1])

$$
\pi_{m n}(g, l)=\mathfrak{\Re}_{m n}(l) u_{m n}(g,-l) \text { for }|m|,|n|=0,1,2, \cdots, l-1
$$

where the $\mathfrak{T}_{m n}(l)$ are nonzero constants. We now have

THEOREM 4.5. Let $W \in E^{\prime}$ be "orthogonal" to the finite dimensional representations of $G$, that is, $W \cdot \pi_{m n}(g, l)=0$ for all $m, n, l$. Then $W=0$.

Proof. Let $m$ and $n$ be fixed. Then by (4.34) we have $W \cdot u_{m n}(g,-l)=0$ for $l \geqq \max (|m|,|n|)$. This means that

$$
\left(\mathfrak{I}_{0} P_{m n} W\right)(l)=0 \text { for } l \geqq \max (|m|,|n|) .
$$

But, $\mathfrak{T}_{0} P_{m n} W$ is an entire function of exponential type which is slowly increasing on $R s=1 / 2$. It follows easily from (4.35) that $\mathfrak{I}_{0} P_{m n} W=0$. (See, e.g. Carleman's formula in Titchmarsh [1, p. 130 or p. 187, no. 7].) Thus, by the corollary to Proposition 4.2, $P_{m n} W=0$. Since this holds for all $m, n$, we have $W=0$ (see Lemma 4.5).

We conclude this section with a description of the center of the algebra $E^{\prime}$ :

CoROlLARY. Let $W \in E^{\prime}$; then $W$ lies in the center of $E^{\prime}$ if and only if

(a) For $m \neq n, P_{m n} W=0$.

(b) For any $i, j, \mathfrak{I}_{0} P_{i i} W=\mathfrak{T}_{0} P_{j j} W$ is a polynomial in $s(1-s)$.

Thus, the center of $E^{\prime}$ consists exactly of the polynomials in $\Delta$. Hence the center of $E^{\prime}$ is identical with the center of the associative enveloping algebra of the Lie algebra of $G$. 
Proof. Suppose first that $W$ satisfies conditions (a) and (b). Then for any $X \in E^{\prime}$, if $\mathfrak{T}_{0} X=\left\{X_{m n}(s)\right\}$, then (writing $W(s)=\mathfrak{T}_{0} P_{i i} W$ ) we have by Proposition 4.5 ,

$$
\mathfrak{T}_{0}(W * X)=\left\{W(s) X_{m n}(s)\right\}=\mathfrak{I}_{0}(X * W) .
$$

It follows immediately from the corollary to Proposition 4.3 and Lemma 4.5 that $W * X=X * W$.

Conversely, suppose that $W$ lies in the center of $E^{\prime}$ and write $\mathfrak{I}_{0} W$ $=\left\{W_{m n}(s)\right\}$. Let $\mathfrak{F}^{i j}$ be the element of $\mathfrak{T}_{0} E^{\prime}$ defined by

$$
F_{m n}^{i j}(s)= \begin{cases}F(s) & \text { if }(i, j)=(m, n), \\ 0 & \text { otherwise }\end{cases}
$$

where $F(s)$ is a suitable nonzero function in $\mathfrak{I}_{0} E_{m n}^{\prime}$.

By our assumption that $W$ lies in the center of $E^{\prime}$ we must have

$$
\left\{W_{m n}(s)\right\} \mathfrak{F}^{i j}=\mathfrak{F}^{i j}\left\{W_{m n}(s)\right\} .
$$

Taking the $r, q$ term of (4.37) we have

$$
W_{r i}(s) F(s) \delta_{q}^{i}=\delta_{r}^{i} F(s) W_{j q}(s) .
$$

If we take $q=j, r \neq i$ in (4.38) then we find

$$
W_{r i}(s) F(s)=0 .
$$

Thus,

$$
W_{r i}(s)=0
$$$$
\text { for } r \neq i \text {. }
$$

Taking $q=j, r=i$ in (4.38) we find

$$
W_{i i}(s)=W_{j j}(s) .
$$

From (4.40) and (4.41) everything follows except the fact that $P_{i i}(s)$ is a polynomial.

Now, let us note that

$$
\omega_{m m}(l)= \begin{cases}1 & \text { if } l \leqq|m|, \\ 0 & \text { if } l>|m| .\end{cases}
$$

It follows immediately from (4.21b) that we must have

$$
W_{l l}(-l)=O\left(1+|l|^{a}\right) \text { for some } a .
$$

Since $W_{l l}(s)=W(s)$ for any $m, l$, this means that $W_{00}(s)$ is slowly increasing on the line $R s=1 / 2$ and on the negative integers. By the functional equation $W_{00}(s)=W_{00}(1-s), W_{00}$ is also slowly increasing on the positive integers. Since $W_{00}$ is an entire function of exponential type, this implies that $W_{00}$ is a polynomial in $s$. 
Since $W_{00}(s)=W_{00}(1-s)$, we may write $W_{00}(s)=Q[(s)(1-s)]$ where $Q$ is a polynomial. It is then clear that $W=Q(\Delta)$.

REMARK. This corollary can be verified directly by noting that the class of conjugate elements of any $g \in G, g \neq 1$, is noncompact, and the fact that any $W$ in the center of $E^{\prime}$ must be invariant under inner automorphism. Thus the support of $W$ must be at $1 \in G$, i.e. $W$ is in the associative enveloping algebra of the Lie algebra of $G$. Hence $W$ is in the center of the associative enveloping algebra which is known to consist of the polynomials in $\Delta$ only.

The noncompactness of the classes of conjugate elements $\not 1(1)$ of $G$ also implies that the center of $D$ consists of 0 only.

It is clear that $\mu_{a}^{(m)}: f \rightarrow F_{m m}(a)$ is for each fixed $m$ and a continuous linear mapping of $D$ to the complex numbers, i.e. an element of $D^{\prime}$. Moreover their sum $\sum \mu_{a}^{(m)}$ converges in the topology of $D^{\prime}$ as follows from the corollary to Theorem 3.3; that is, the series $\sum_{m} u_{m m}(g, a)$ converges for each fixed $a$ in the topology of $D^{\prime}$. In this sense the "group-characters" exist. (The existence of group-characters as distributions on semi-simple Lie groups is of course due to Harish-Chandra.) We thus obtain an element of $D^{\prime}$ which we denote by $\chi_{a}$ :

$$
\chi_{a}(f)=\sum_{m=-\infty}^{\infty} F_{m m}(a)
$$

It is readily verified that $\chi_{a}$ is invariant under inner automorphism of $G$ and is independent of the particular complete orthonormal system of $L_{2}((0,1)$, $d \theta)$.

LEMMA 4.6. $\mathfrak{T}_{0} \chi_{a}=\delta_{a}$ in the sense that $\mathfrak{T}_{0} \chi_{a} \cdot X(s)=$ trace of $X(a)$ for any $X(s) \in \mathfrak{T}_{0} D$.

Proof. It follows at once that $\mathfrak{I}_{0} \mu_{a}^{m} \cdot X(s)=X_{m m}(a)$ hence $\sum_{m} \mu_{a}^{(m)} \cdot X(s)$ $=\sum_{m} X_{m m}(a)$ for any $X(s) \in T_{0} D$.

Note that the restriction of $\chi_{s}$ to $P_{m n} D$ equals $\delta_{m n} \mu_{s}^{(m)}$, i.e. $P_{m n} \chi_{s}=\delta_{m n} \mu_{s}^{(m)}$. Thus

$$
\begin{aligned}
P_{m m} \chi_{s} \cdot f & =\chi_{s}\left(P_{m m} f\right)=\int_{G}\left(P_{m m} f\right)(g) u_{m m}(g, s) d g \\
& =\int_{G} f(g) u_{m m}(g, s) d g .
\end{aligned}
$$

In particular

$$
P_{00} \chi_{s} \cdot f=\int_{G} f(g) \phi(g, s) d g
$$

establishes a one-one correspondence between the characters $\chi_{s}$ and the elementary spherical functions $\phi(g, s)$, as is easily verified. 
5. Mean periodic functions of fixed type $m, n$. In this section we shall carry over the theory of mean periodic functions of L. Schwartz [3] to spherical functions of fixed type $m, n$ on $G$. We shall obtain the fundamental theorem for mean periodic functions and also an analog of the formal expansion of mean periodic functions.

Let $f \in E$. Then it would seem natural to say that $f$ is (left) mean periodic if there exists an $a \in E^{\prime}, a \neq 0$, such that $a * f=0$. However, then every $f \in E_{m n}$ would be left mean periodic because any $a \in E_{j p}^{\prime}$ for $p \neq m$ satisfies $a * f=0$.

A second possibility for defining mean periodicity is the following: a function $f \in E$ is left mean periodic if the set $\{a * f\}_{a \in E^{\prime}}$ is not dense in $E$; $f$ is right mean periodic if the set $\left\{f * b, b \in E^{\prime}\right\}$ is not dense in $E$. A function is mean periodic if it is both left and right mean periodic. This definition turns out to be suitable in the general case. However, for $f(g) \in E_{m n}$ we shall use a different definition for convenience. We shall show (Proposition 5.2 below) that both definitions are equivalent.

Definition 5.1. A function $f(g) \in E_{m n}$ is called right mean periodic if there exists an integer $j$ and a $W \in E_{j_{m}}^{\prime}, W \neq 0$, such that $W * f=0$. Left mean periodicity is defined similarly, and $f(g)$ is said to be mean periodic if it is both left and right mean periodic.

Proposition 5.1. Suppose $f(g) \in E_{m n}$ is right mean periodic. Then for any $i$ there is an $h \in D_{i m}, h \neq 0$, such that $h * f=0$. A similar property holds for left mean periodicity.

Proof. We know that for some $j$ we can find a $W \in E_{j_{m}}^{\prime}, W \neq 0$ such that $W * f=0$. Let $t \in D_{i j}, t \neq 0$. By Theorem 4.1, Proposition 4.3, and Theorem 2.1 , we have $t * W \in D_{i m}, t * W \neq 0$. Thus,

$$
0=t *(W * f)=(t * W) * f
$$

which is the desired result. The part of Proposition 5.1 dealing with left mean periodicity is proved similarly.

Proposition 5.2. Let $f(g) \in E_{m n}$. A necessary and sufficient condition that $f(g)$ be left mean periodic is that the set

$$
\Omega_{j}\left\{P_{j m} a * f ; a \in E^{\prime}\right\}
$$

be not dense in $E_{j m}$ for some $j$. A similar property holds for right mean periodicity.

Proof. Assume first that $\Omega_{r}$ is not dense in $E_{r m}$. By the Hahn-Banach theorem, we can find a $W \in E_{r n}^{\prime}, W \neq 0$, such that $W \cdot(a * f)=0$ for all $a \in E_{r n}$. Now, we have

$$
W \cdot a * f=(\widetilde{a})^{*} \cdot W * f=(\breve{a})^{*} \cdot f *(\widetilde{W})^{*}
$$

(where $(\widetilde{a})^{*}(g)=\bar{a}\left(g^{-1}\right)$ is identified with a distribution by means of equation 
(4.2)). Thus, by the generalized Hahn-Banach theorem, $f^{*}(\widetilde{W})^{*}=0$. But, clearly, $(\widetilde{W})^{*} \in E_{n r}^{\prime}$, so that $f$ is left mean periodic.

Conversely, suppose $f(g)$ is left mean periodic, so for some $r$ there exists a $W \in E_{r n}^{\prime}, W \neq 0$, such that $f *(\widetilde{W})^{*}=0$. Then for any $a \in E_{r m}$,

$$
W \cdot a * f=(\widetilde{a})^{*} \cdot f *(\widetilde{W})^{*}=0
$$

by (5.2). Thus, $\Omega_{r}$ cannot be dense in $E_{r m}$.

In establishing the analog of the fundamental theorem of mean periodic functions of L. Schwartz [3], we shall show that every mean periodic function possesses a "spectrum." Moreover, if $f$ is left (right) mean periodic, then it possesses a left (right) spectrum. We shall show, in fact, that both left and right mean periodicity are the same and that the left and right spectra are the same.

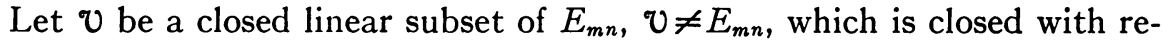
spect to left convolution by functions of $D_{m m}$. Then we call $v$ a (left) variety. Since $\mathcal{V} \neq E_{m n}$, there is a $W \in E_{m n}^{\prime}$ such that $W \cdot f=0$ for all $f \in \mathcal{V}$. Hence also $W \cdot a * f=0$ for all $f \in \mathcal{V}, a \in E_{m m}^{\prime}$. Now, by (5.2),

$$
W \cdot a * f=(\breve{a})^{*} \cdot f *(\widetilde{W})^{*}=0
$$

for all $a \in E_{m m}^{\prime}$. By the generalized Hahn-Banach theorem, we must have $f *(\overleftarrow{W})^{*}=0$. Thus, for any $X \in E_{m n}^{\prime}, f \in \mathcal{V}$, we have $f *\left(\left(\breve{W}^{*} * X\right)=0\right.$. By Theorem $4.1,(\widetilde{W})^{*} * X \neq 0$. Thus, we conclude the existence of some $Y \in E_{n n}^{\prime}$, $Y \neq 0$ with $f * Y=0$ for all $f \in v$.

By $\mathcal{V}^{\prime}$ we denote the set of all $Y \in R_{n n}^{\prime}$ which satisfy $f * Y=0$ for all $f \in \mathcal{V}$. It is clear that $\mathcal{V}^{\prime}$ is a closed ideal in $E_{n n}^{\prime}$. (Since $E_{n n}^{\prime}$ is a commutative algebra, we need not distinguish between left and right.) We call $V^{\prime}$ the annihilator ideal of $v$.

Conversely, given any ideal $\mathscr{g}$ in $E_{n n}^{\prime}$, and any $m$, denote by $\mathscr{I}_{m}^{\prime}$ the set of all $f \in E_{m n}$ which satisfy $f * W=0$ for all $W \in g$. It follows easily that $\mathscr{g}_{m}^{\prime}$ is a variety, which we call the $(m)$-annihilator variety of $g$.

Proposition 5.3. For any variety $v$ in $E_{m n}$, call $v^{\prime \prime}$ the $(m)$-annihilator variety of $V^{\prime}$. Then $V^{\prime \prime}=\mathcal{V}$. For any closed ideal $g$ in $E_{n n}^{\prime}, \mathfrak{I}_{m}^{\prime \prime}=\mathfrak{g}$.

Proof. (a) It is clear that $\mho^{\prime \prime} \supset \mho$; assume $f \in V^{\prime \prime}, f \in v$. By the generalized Hahn-Banach theorem we can find a $W \in E_{m n}^{\prime}$ such that

$$
W \cdot f=1, \quad W \cdot h=0 \text { for all } h \in v .
$$

Since $\mathcal{V}$ is a variety, $P_{m m} \delta_{0} * h \in \mathcal{V}$ for all $g \in G$. But, for any $g \in G$,

$$
W \cdot P_{m m} \delta_{o} * h=W \cdot P_{m n}\left(\delta_{g} * h\right)=W \cdot\left(\delta_{\theta} * h\right)=(h *(\overleftarrow{W}))^{*}\left(g^{-1}\right)=0
$$

by (5.2) and (5.3).

Hence, for any $X \in E_{m n}^{\prime},(\widetilde{W})^{*} * X \in \mathcal{V}^{\prime}$. By our assumptions on $f$, we must have $f *(\widetilde{W})^{*} * X=0$. Thus, for any $Y \in E_{m n}^{\prime}$, we have 


$$
\begin{aligned}
0 & =\left(f *(\widetilde{W})^{*} * X *(\widetilde{Y})^{*}\right)(1) \\
& =\left(X *(\widetilde{Y})^{*}\right) \cdot\left(f *(\widetilde{W})^{*}\right) .
\end{aligned}
$$

It follows easily from Theorem 4.2 that every $Z \in E_{m m}^{\prime}$ is of the form $X *(\breve{Y})$ * for $X, Y \in E_{m n}^{\prime}$. Thus, by (5.5) and the generalized Hahn-Banach theorem, $f *(\widetilde{W})^{*}=0$. In particular,

$$
0=(f * \widetilde{W})(1)=W \cdot f .
$$

This contradicts (5.4) and so concludes the proof of the first part of Proposition 5.3.

(b) The proof of the second part is similar.

Proposition 5.3 shows us that any problem about varieties can be translated into an equivalent problem on its annihilator ideal. By Theorem 4.1, this problem is translated into a problem about the ring $\mathfrak{T}_{0} E_{n n}^{\prime}$ of entire functions of exponential type which are slowly increasing on $R s=1 / 2$ and are invariant under the transformation $s \rightarrow 1-s$ (see Theorem 4.1).

For future reference we shall need the following result, the proof of which follows without difficulty from the results of J. Dieudonné and L. Schwartz [1]:

Proposition 5.4. Let $\mathfrak{H}$ be a complete, metrizable, locally convex topological vector space, and denote by $\mathcal{H}^{\prime}$ the dual of $\mathcal{H C}$ with the topology of uniform convergence on the bounded sets of $\mathcal{H}$. Then every sequentially closed linear subset of $H^{\prime}$ is closed.

For any $s_{0}, m, n$, we may identify $u_{m n}\left(g, s_{0}\right)$ with a distribution on $G$ by means of 4.2 . Thus, by the method of $\S 4, \mathfrak{I}_{0} u_{m n}\left(g, s_{0}\right)$ is defined. Since $\mathfrak{I}_{0}$ is one-one on $D$, we can consider $\mathfrak{T}_{0} u_{m n}\left(g, s_{0}\right)$ as a continuous linear function on $\mathfrak{T}_{0} D$.

Proposition 5.5. For any $f \in D$, where $\mathfrak{T}_{0} f=\left(F_{m n}(s)\right)$,

$$
\left[\mathfrak{T}_{0} u_{m n}\left(g, s_{0}\right)\right] \cdot\left[\mathfrak{T}_{0} f\right]=F_{-m,-n}\left(\bar{s}_{0}\right) .
$$

Proof. By definition,

$$
\left[\mathfrak{T}_{0} u_{m n}\left(g, s_{0}\right)\right] \cdot\left[\mathfrak{T}_{0} f\right]=u_{m n}\left(g, s_{0}\right) \cdot f=\int f(g) \bar{u}_{m n}\left(g, s_{0}\right) d g=F_{-m,-n}\left(\bar{s}_{0}\right)
$$

because (as follows easily from the definitions) $\bar{u}_{m n}\left(g, s_{0}\right)=u_{-m-n}\left(g, \bar{s}_{0}\right)$.

We shall write $\mathfrak{I}_{0} u_{m n}\left(g, s_{0}\right)=\delta_{s_{0}}^{-m,-n}$ so $\delta_{s_{0}}^{-m_{1}-n}$ is, when restricted to $\mathfrak{T}_{0} D_{m n}$ (or $\left.\mathfrak{T}_{0} E_{m n}^{\prime}\right)$, the unit mass at the "point" $\left(\bar{s}_{0},-m,-n\right)$. We shall write, for any $j \geqq 0$,

$$
u_{m n}^{(j)}\left(g, s_{0}\right)=\mathfrak{T}^{-1}\left(\frac{d^{j}}{d s^{j}} \delta_{s_{0}}^{-m,-n}\right) .
$$


Clearly, $u_{m n}^{(0)}\left(g, s_{0}\right)=u_{m n}\left(g, s_{0}\right)$, and it is not difficult to show that, in general, $u_{m n}^{(j)}\left(g, s_{0}\right)=\left(d^{i} / d s^{i}\right) u_{m n}\left(g, s_{0}\right)$.

Proposition 5.6. Let $\mathfrak{g}$ be a closed left ideal in $E_{m m}^{\prime}$, and set $\mathcal{g}=\mathfrak{g} \cap D_{m m}$ so $\mathcal{J}$ is an ideal in $D_{m m}$. Then $\mathcal{J}$ determines $\mathfrak{g}$ and $\mathfrak{g}$ is the closure of $\mathcal{g}$ in $E_{m m}^{\prime}$.

Proof. Denote by $\bar{g}$ the closure of $\mathfrak{g}$ in $E_{m m}^{\prime}$. Now, for any $W \in \mathfrak{g}, W$ $=\lim _{E_{m m}^{\prime}} f_{j} * W$ where $\left\{f_{j}\right\}$ is a sequence in $D_{m m}$. But, since $g$ is a closed left ideal, $f_{j} * W \in g$; on the other hand, we clearly have $f_{j} * W \in D_{m m}$. This proves that $\mathfrak{g} \subset g$; the reverse inclusion is obvious.

Proposition 5.7. Let $v$ be a left variety in $E_{m n}$. Then $u_{m n}^{(j)}\left(g, s_{0}\right) \in \mathcal{V}$ if and only if $\mathfrak{I}_{0} \mathcal{V}^{\prime}$ has a zero of order $j+1$ at $s_{0}$. If $u_{m n}^{(j)}\left(g, s_{0}\right) \in \mathcal{V}$ then also $u_{m n}^{(i)}\left(g, s_{0}\right)$ $\in \mathcal{V}$ whenever $0 \leqq i \leqq j$.

Proof. Suppose first that $u_{m n}^{(j)}\left(g, s_{0}\right) \in \mathcal{V}$. Then for any $f \in \mathcal{V}^{\prime} \cap D$,

$$
\begin{aligned}
0 & =\left[u_{m n}^{(j)}\left(g, s_{0}\right) * f * W\right](1)=\bar{u}_{m n}^{(j)}\left(g, s_{0}\right) \cdot[f * W] \\
& =\frac{d^{i}}{d s^{j}}\left[\mathfrak{T}_{0}\left(f^{i} W\right)\right]\left(s_{0}\right)=\frac{d^{j}}{d s^{j}}\left[\left(\mathfrak{T}_{0} f\right)\left(\mathfrak{T}_{0} W\right)\right]\left(s_{0}\right) .
\end{aligned}
$$

From (5.10) and Theorem 4.1 we deduce easily that $\left[d^{i}\left(\mathfrak{T}_{0} f\right) / d s^{i}\right]\left(s_{0}\right)=0$ for $0 \leqq i \leqq j$. By Proposition 5.6, $\left[d^{i}\left(\mathfrak{T}_{0} X\right) / d s^{i}\right]\left(s_{0}\right)=0$ for any $X \in \mathcal{V}^{\prime}$, $0 \leqq i \leqq j$.

Conversely, suppose that $s_{0}$ is a $j+1$-fold zero of $\mathfrak{I}_{0} \mathcal{V}^{\prime}$. Then for $0 \leqq i \leqq j$, and any $f \in V^{\prime} \cap D, x \in G$,

$$
\begin{aligned}
{\left[u_{m n}^{(i)}\left(g, s_{0}\right) * f\right](x) } & =\bar{u}_{m n}^{(i)}\left(g, s_{0}\right) \cdot L(x)[f * W] \\
& =\frac{d^{i}}{d s^{i}}\left[\mathfrak{T}_{0} P_{m n} L(x)(f * W)\right]\left(s_{0}\right) \\
& =\left.\frac{d^{i}}{d s^{i}} \int u_{m n}(g, s) f\left(g^{-1} x\right) d g\right|_{s=s_{0}} \\
& =\left.\frac{d^{i}}{d s^{i}} \int u_{m n}\left(x y^{-1}, s\right) f(y) d y\right|_{s=s_{0}} \\
& =\left.\frac{d^{i}}{d s^{i}} \int \sum_{l} u_{m l}(x, s) u_{l n}\left(y^{-1}, s\right) f(y) d y\right|_{s=s_{0}} \\
& =\left.\frac{d^{i}}{d s^{i}} \sum_{l} \int u_{m l}(x, s) u_{l n}\left(y^{-1}, s\right) f(y) d y\right|_{s=s_{0}} \\
& =\left.\frac{d^{i}}{d s^{i}}\left[u_{m n}(x, s) F(s)\right]\right|_{8-s_{0}}
\end{aligned}
$$


where $F(s)=\left(\mathfrak{T}_{0} f\right)(s)$ and where the order of integration and summation may be inverted by Theorem 3.1 .

We conclude that

$$
u_{m n}^{(i)}\left(g, s_{0}\right) * f=0 \quad \text { for } 0 \leqq i \leqq j
$$

which, together with Proposition 5.7, implies $u_{m n}^{(i)}\left(g, s_{0}\right) \in \mathcal{V}$ for $0 \leqq i \leqq j$. The last statement of Proposition 5.6 is immediate.

COROllary. If $i<j, u_{m n}^{(i)}\left(g, s_{0}\right)$ is the limit of left (or right) convolutions of $u_{m n}^{(f)}\left(g, s_{0}\right)$.

Let $v$ be a left variety in $E_{m n}$. By the (left) spectrum of $v$ (or the cospectrum of $\left.\mathcal{U}^{\prime}\right)$ we mean the set of pairs $\left(s_{0}, j\right)$ where $u_{m n}^{(i)}\left(g, s_{0}\right) \in V$ for $0 \leqq i \leqq j-1$. By Proposition 5.7 this is the same as the set of pairs $\left(s_{0}, j\right)$ where $s_{0}$ is a $j$-fold zero of $\mathfrak{I}_{0} \mathcal{U}^{\prime}$.

Let $\mathscr{g}$ be a closed ideal in $E_{m m}^{\prime}$, and set $\mathfrak{J}=\mathfrak{T}_{0} \mathscr{g}$, so $\mathcal{g}$ is a closed ideal in $\mathfrak{T}_{0} E_{m m}^{\prime}$; the latter is the ring of all entire functions of exponential type which satisfy $F(s)=F(1-s)$ and are slowly increasing on the line $R s=1 / 2$. We shall show that $\mathcal{I}$ has a zero and is, in fact, determined by the cospectrum of $\mathfrak{g}$ (which we shall also call the cospectrum of $\mathrm{g}$ ).

Lemma 5.1. $\mathcal{J}$ is a closed subset of $\widehat{\mathcal{E}}^{\prime}$. Denote by $\tilde{\mathfrak{J}}$ the ideal generated by $\mathrm{J}$ in $\widehat{\mathcal{E}}^{\prime} ;$ then $\tilde{\mathfrak{J}} \cap E_{m m}^{\prime}=\mathcal{J}$.

Proof. We know that $g$ is sequentially closed in $E_{m m}^{\prime}$. Hence by Theorem $4.3, g$ is sequentially closed in $E^{\prime}$. By Proposition $5.4, g$ is closed in $E^{\prime}$.

Now, $\tilde{g}$ is, by Proposition 5.4, the sequential closure of the algebraic ideal generated by $\mathcal{g}$ in $\widehat{\mathcal{E}}^{\prime}$. Thus, if $H \in \tilde{\mathcal{J}} \sim E_{m m}^{\prime}$, we can find sequences $\left\{F_{l}\right\} \subset E^{\prime}$, $\left\{H_{l}\right\} \subset J$ such that $F_{l} H_{l} \rightarrow H$ in the topology of $\widehat{\mathcal{E}}^{\prime}$. We write

$$
\widetilde{F}_{l}(s)=\left(F_{l}(s)+F_{l}(1-s)\right) / 2,
$$

so also $\widetilde{F}_{l} H_{l} \rightarrow H$ in $\widehat{\mathcal{E}}^{\prime}$. But, $\widetilde{F}_{l} \in E_{m m}^{\prime}$. Thus, by Theorem 4.3, $\widetilde{F}_{l} H_{l} \rightarrow H$ in the topology of $\mathfrak{T}_{0} E^{\prime}$. This means that $H \in \mathcal{d}$, i.e. $\tilde{\mathfrak{g}} \cap E_{m m}^{\prime} \subset \mathcal{d}$. The result is now immediate.

THEOREM 5.1. Every closed left-module $\mathcal{S} \subset \mathfrak{T}_{0} E_{m n}^{\prime}$ over $\mathfrak{T}_{0} E_{m m}^{\prime}$ is determined by its cospectrum. That is, if $F(s) \in \mathfrak{T}_{0} E_{m n}^{\prime}$ vanishes on the zeros of $\mathrm{d}$ with correct multiplicities, then $F(s) \in \mathcal{J}$. A similar result holds for $D_{m n}$ in place of $E_{m n}^{\prime}$.

Proof. This result is an immediate consequence of Lemma 5.1 and the corresponding result for the space $\mathcal{E}^{\prime}$ of Schwartz on the real line and Propositions 5.7 and 5.6.

As an immediate consequence of Theorem 5.1 we have

Corollary. For fixed $m, n$, the maximal closed left-modules of $D_{m n}$ over $D_{m m}$ are given as follows:

If $m=n$ then each maximal closed ideal of the algebra $D_{m m}$ is determined by a 
complex number $s_{0}$ and is the set $g_{s_{0}}$ all $f(g) \in D_{m m}$ for which $\left(\mathfrak{T}_{0} f\left(s_{0}\right)=0\right.$.

If $m \neq n$ then any complex number $s_{0}$ determines a maximal closed leftmodule $\mathcal{d}_{s_{0}}$ consisting again of all $f \in D_{m n}$ for which $\left(\mathfrak{T}_{0} f\right)\left(s_{0}\right)=0$, unless $\left(\mathfrak{I}_{0} D_{m n}\right)\left(s_{0}\right)=(0)$. For the remaining values of $s_{0}$ we obtain a maximal module $\mathcal{d}_{s_{0}}$ consisting of all $f \in D_{m n}$ for which $d\left(\mathfrak{T}_{0} f\right)\left(s_{0}\right) / d s=0$. These are the only maximal modules. Moreover $\mathcal{d}_{s}=J_{s^{\prime}}$ if and only if $s=s^{\prime}$ or $s=1-s^{\prime}$. The same result holds for $E_{m n}^{\prime}$ in place of $D_{m n}$.

From Theorem 5.1, Propositions 5.3 and 5.7 we deduce

Theorem 5.2. Let $v$ be a left-variety in $E_{m n}, v \neq(0)$. Then $v$ contains $a$ function $u_{m n}\left(g, s_{0}\right)$ for some $s_{0}$. Moreover $v$ is completely determined by the functions $u_{m n}^{(j)}(g, s)=\partial^{j} u_{m n}\left(g, s_{l}\right) / \partial s^{i}$ which it contains. Moreover no $u_{m n}^{(j)}\left(g, s_{l}\right)$ $\in \mathcal{V}$ is the limit of linear combinations of $u_{m n}^{(i)}\left(g, s_{r}\right) \in \mathcal{V}$ for $s_{r} \neq s_{l}$ and any $i$, or for $s_{r}=s_{l}$ and $i<j$. Finally, $v$ is the closed linear subspace generated in $E_{m n}$ by those functions $u_{m n}^{(p)}\left(g, s_{i}\right)$ of $g$ which are contained in $v$.

CoRollary 1. Every left variety in $E_{m n}$ is also a right variety. The left spectrum and right spectrum of any variety $v$ are identical.

CoROllary 2. Every left (right) mean periodic function of type $m, n$ is mean periodic.

Let $f(g) \in E_{m m}, f \neq 0$, be mean periodic; denote by $v$ the variety generated by $f(g)$ in $E_{m m}$, and write $\mathfrak{g}=\mathfrak{I}_{0} \mathcal{V}^{\prime}\left(\mathcal{V}^{\prime}=\right.$ left annihilator ideal of $\left.\mathcal{V}\right)$. Let us consider $E_{m m}$ as a subspace of $D_{m m}^{\prime}$, using the convention (4.2). We have defined the Fourier transform $\mathfrak{I}_{0}$ on $D^{\prime}$ in $\S 4$; thus $\mathfrak{I}_{0} f(g)$ is defined for $f \in E_{m m}$. Since $\mathfrak{T}_{0}$ is one-one, we may consider $\mathfrak{T}_{0} f$ as a linear function on $\mathfrak{T}_{0} D_{m m}$, and. in fact, as a linear function on $\mathfrak{T}_{0} E_{m m}^{\prime}$. Now, define, for any $H(s) \in \widehat{\mathcal{E}}^{\prime}$,

$$
\mathfrak{T}_{0} f \cdot H(s)=\mathfrak{T} f \cdot[(H(s)+H(1-s)) / 2] .
$$

Thus, by Theorem 4.3, $\mathfrak{I} f$ is bounded on the bounded sets of $\widehat{\mathcal{E}}^{\prime}$. We have already remarked that $\widehat{\mathcal{E}}^{\prime}$ is bornologic, so $\mathfrak{T} f$ defines an element of $\widehat{\mathcal{E}}$, the dual of $\widehat{\mathcal{E}}^{\prime}$; we denote this element by $X$. It is clear that $X$ vanishes on $\tilde{\boldsymbol{g}}$, the closed ideal generated by $g$ in $\hat{E}^{\prime}$. Moreover, if $\mathcal{g}$ denotes the largest closed ideal on which $X$ vanishes (this clearly exists) then we must have $\mathfrak{g} \cap \mathfrak{T}_{0} E_{m m}^{\prime}=\mathfrak{g}$, because $\mathfrak{g} \cap \mathfrak{T}_{0} E_{m m}^{\prime}$ is an ideal in $\mathfrak{T}_{0} E_{m m}^{\prime}$ (by Proposition 5.4 and Theorem 4.3) on which $\mathfrak{I} f$ vanishes. Since every ideal in $\mathcal{E}^{\prime}$ is determined by its cospectrum (see Schwartz [3]) it follows that $\mathfrak{g}=\tilde{g}$.

Thus, according to the result of Schwartz [3] we can find numbers $d_{p q}$, $q=0,1, \cdots, r$, for each point $s_{p}$ on which $\tilde{g}$ vanishes to order $r+1$, where

$$
\sum_{p, q} d_{p q} \delta_{p}^{(q)}
$$

converges to $X$ in $\widehat{\varepsilon}$ by means of grouping of terms and Abel convergence 
factors, and where $\delta_{p}^{q} \cdot H(s)=\left(d^{q} H / d s^{q}\right)\left(s_{p}\right)$. Now, $\widehat{\mathcal{E}}$ and $\mathfrak{T}_{0} E_{m m}$ have the topologies defined by uniform convergence on bounded sets of $\widehat{\mathcal{E}}^{\prime}$ and $\mathfrak{I}_{0} E_{m m}^{\prime}$ respectively. Thus, by Theorem 4.3 , the topology induced by $\widehat{\mathcal{E}}$ on $\mathfrak{T} E_{m m}$ is stronger than (it is easily seen to be the same as) the topology of $\mathfrak{I}_{0} E_{m m}$. We conclude that

$$
\sum_{p, q} d_{p q} \delta_{p}^{(b)} \text { converges to } \mathfrak{T}_{0} f \text { in } \mathfrak{T}_{0} E_{m m}
$$

by means of grouping of terms and Abel convergence.

A similar argument works for $E_{m n}$, except that we must consider $E_{m n}$ as a module over $E_{m m}$. The right side of (5.11) must be replaced by

$$
T f \cdot\left[\eta_{m n}(s)(H(s)+H(1-s)) / 2\right]
$$

where $\eta_{m n}(s)$ is defined as in the proof of Theorem 2.1.

Applying the inverse Fourier transform $\mathfrak{T}_{0}^{-1}$ we then deduce:

THEOREM 5.3. Let $f(g) \in E_{m n}$ be mean periodic. Then we can find:

(1) a sequence of pairs $\left(s_{p}, q\right)$ where $s_{p}$ are complex numbers, and, for each $p, q$ runs over a finite set of non-negative integers;

(2) a sequence of complex numbers $d_{p q}$ for any $\left(s_{p}, q\right)$ of (1) such that

$$
\sum_{p, q} d_{p q} u_{m n}^{(q)}\left(g, s_{p}\right) \text { converges to } f(g) \text { in } E_{m n}
$$

by means of grouping of terms and Abel convergence factors.

The functions $u_{m n}^{(e)}\left(g, s_{p}\right)$ of $g$ which occur in (5.13) are contained in the variety generated by $f(g)$ in $E_{m n}$.

We say that the series $\sum d_{p q} u_{m n}^{(q)}\left(g, s_{p}\right)$ converges to $f(g)$ in $E_{m n}$ by means of groupings of terms and Abel convergence factors (see Schwartz [2;3]) if we can find a partition of the set of all pairs of positive integers into disjoint subsets $\#_{j}$ such that for each $y>0$ the series

$$
\sum_{j}\left[\sum_{p, q \in \#_{j}} d_{p q} u_{m n}^{(q)}\left(g, s_{p}\right) \exp \left(-\left|R\left(s_{p}-\frac{1}{2}\right)\right| y\right)\right]
$$

converges in the topology of $E_{m n}$ to a function $f_{y}(g)$ for which $\lim _{y \rightarrow 0} f_{y}=f$ in the topology of $E_{m n}$.

6. Two-sided mean periodic functions in $E$. In the previous section we studied mean periodic spherical functions of fixed type $m, n$. We shall now consider another notion of mean periodicity which is not restricted to spherical functions.

Definition 6.1. Let $f(g) \in E$. We denote by $M_{f}$ the closure in $E$ of the set $D * f * D$. The function $f(g)$ will be called two-sided mean periodic if $M_{f} \neq E$.

Definition 6.2. Let $M$ be a closed two-sided submodule of $E$ over $D$. By $M^{1}$ we denote the set of all $w \in E^{\prime}$ such that $w * M=M * w=(0)$. Let $J$ 
be a closed two-sided ideal of $E^{\prime}$. By $J^{1}$ we denote the set of all $h \in E$ such that $h * J=J * h=(0)$.

Lemma 6.1. Let $V$ be a closed linear subspace of $E$. Then $L_{g} V \subseteq V$ for all $g \in G$ is equivalent to $D * V \subseteq V$ and is also equivalent to $E^{\prime} * V \subseteq V$. Similarly for right translation $R_{g}$ and convolution on the right.

Lemma 6.1 follows in the usual manner from the standard properties of convolution.

LemMA 6.2. $M^{1}$ is a closed two-sided ideal of $E^{\prime}$ and $J^{1}$ is a closed two-sided module in $E$ over D. Moreover $\left(M^{1}\right)^{1}=M$ and $\left(J^{1}\right)^{1}=J$.

Proof. From the continuity of convolution and the closure of $M$ it follows that $M^{1}$ is a closed two-sided ideal of $E^{\prime}$. It is clear that $\left(M^{1}\right)^{1} \supseteq M$. Now assume there exists an element $h$ of $\left(M^{1}\right)^{1}$, but $h \notin M$. Then by the generalized Hahn-Banach theorem there exists an element $w$ of $E^{\prime}$ such that $w(h) \neq 0$, but $w(M)=(0)$. It follows from Lemma 6.1 that $w * M=(0)=M * w$, thus $w \in M^{1}$. Also $w(h) \neq 0$ implies $w * h \neq 0$. But $h \in\left(M^{1}\right)^{1}$ and $w \in M^{1}$ imply $h * w=0$. This contradiction proves $\left(M^{1}\right)^{1}=M$.

The assertions about $J$ follow in the same way.

Leмma 6.3. Let $J \neq(0)$ be a closed two-sided ideal in $E^{\prime}$. Then $P_{m n} J \neq(0)$ for any integers $m, n$.

Proof. Assume there exist integers $m, n$ such that $J_{m n}=P_{m n} J=(0)$. Let $p, q$ be arbitrary integers. Then $E_{m p *}^{\prime} J_{p q *} E_{q n}^{\prime} \subseteq J_{m n}$.

By $\S \S 4$ and 5, $a \in E_{m p}^{\prime}$ and $w \in E_{p q}^{\prime}$ imply $a * w \neq 0$ provided $a \neq 0$ and $w \neq 0$. This is seen by taking the Fourier transform of $a, w$ and $a * w$ : one obtains the product of two entire functions which is not identically zero unless one factor is. Choose $a \in E_{m p}^{\prime}, b \in E_{n q}^{\prime}$ so that $a \neq 0$ and $b \neq 0$. Since $J \neq(0)$ there exists a pair $p, q$ such that $P_{p q} J=J_{p q} \neq(0)$. So let $w \in J_{p q}, w \neq 0$. Then by the above $a * w * b \neq 0$ and $a * w * b \in J_{m n}$. This proves that $J_{m n} \neq(0)$.

Leмma 6.4. Let $M$ be a closed two-sided module in $E$ over $D$. If $M \neq E$ then $P_{m n} M \neq E_{m n}$ for any $m, n$.

Proof. By Lemma 6.2, $M \neq E$ is equivalent to $M^{1} \neq(0)$. So there is an element $w \in M^{1}$ and $w \neq 0$. This means $M * w=(0)=w * M$. Moreover $P_{m n}\left(M^{1}\right) \subseteq M^{1}$. By Lemma 6.3, $P_{m n}\left(M^{1}\right) \neq(0)$ for any $m, n$. Thus for any integers $\mu, m, n$ and $\nu$ there exist elements $w_{\mu m} \in E^{\prime}$ and $w_{n \nu} \in E_{n \nu}^{\prime}$ such that $w_{\mu m} \neq 0, w_{n \nu} \neq 0$ but $w_{\mu m} * h=0$ and $h * w_{n \nu}=0$ for $h \in M$. Writing again $P_{m n} h=h_{m n}$ we obtain $w_{\mu m} * h_{m n}=0$ and $h_{m n} * w_{n \nu}=0$ for all $h_{m n} \in P_{m n} M$. This proves that $P_{m n} M \neq E_{m n}$ for any $m, n$.

Theorem 6.1. Let $M$ be any closed proper two-sided module in $E$ over $D$. Then $M$ is the smallest closed linear subspace in $E$ which contains all 


$$
\frac{\partial^{j}}{\partial s^{j}} u_{m n}(g, s) \in M
$$

Proof. For fixed $m, n$ we know by Theorem 5.2 that $M_{m n}=P_{m n} M$ is the smallest closed linear subspace in $E_{m n}$ which contains all $u_{m n}^{(j)}(g, s) \in E_{m n}$, since $M_{m n}$ is a left-module over $D_{m m}$ whenever $M$ is a $D$-module. Now we use Lemma 4.5 and see that $M$ is the smallest closed linear subspace of $E$ containing all $P_{m n} M$.

Lemma 6.5. Let $f \in E$ be two-sided mean periodic in the sense of Definition 6.1. Then $P_{m n} f$ is for each $m, n$ mean periodic in the sense of $\$ 5$.

Proof. This is an immediate consequence of Lemma 6.4.

We can combine Lemma 6.5 and Theorem 5.3 to obtain an expansion theorem for two-sided mean periodic functions in $E$. Indeed let $f \in E$ be twosided mean periodic in the sense of Definition 6.1. Then by Lemma 6.5 $f_{m n}=P_{m n} f$ is a mean periodic spherical function of type $m, n$ in the sense of Definition 5.1, to which we can apply the expansion of Theorem 5.3. Now apply Lemma 4.5 . This proves

TheOREm 6.2. Let $f(g) \in E$ be two-sided mean periodic in the sense of Definition 6.1 above. Then

$$
f(g)=\sum_{m n}\left\{\sum_{p, q} d_{p q} u_{m n}^{(q)}\left(g, s_{p}\right)\right\}
$$

where $\sum_{p, q}$ is in the sense of the summability by grouping of terms and Abel convergence factors as explained at the end of $\$ 5$ and $\sum_{m, n}$ converges in the topology of $E$.

\section{BIBLIOGRAPHY}

\section{BARGMANN}

1. Unitary representations of the Lorentz group, Ann. of Math. vol. 48 (1947) pp. 568-640. N. BOURBAKI

1. XV, Livre V. Espaces vectoriels topologiques, Paris, Hermann, 1953.

2. XIII, Livre 6. Integration, Chapter III. Paris, Hermann, 1953.

L. DeLsarte

1. Sur une extension de la formule de Taylor, J. Math. Pures Appl. vol. 17 (1938).

J. Dieudonne and L. Schwartz

1. La dualité dans les espaces $(F)$ et $(L F)$. Ann. Institut Fourier (Grenoble) vol. 1, (1950) pp. 61-101.

L. EHRENPREIS

1. Theory of distributions for locally compact spaces, Memoirs of the American Mathematical Society, no. 21, 1956.

2. Some properties of distributions on Lie groups (to appear in Pacific Journal of Mathematics).

3. Solutions of some problems of division I, Amer. J. Math. vol. 76 (1954) pp. 883-903.

4. Analytic functions and the Fourier transform of distributions I, Ann. of Math. vol. 63 (1956) pp. 129-159. 
5. Mean periodic functions I, Amer. J. Math. vol. 78 (1955) pp. 293-328.

L. EHRENPREIS and F. I. MAUTNER

1. Some properties of the Fourier transform on semi-simple Lie groups. I, Ann. of Math. vol. 61 (1955) pp. 406-439.

A. Grothendieck

1. Sur les espaces $(F)$ et $(D F)$, Summa Brasiliensis Mathematicae vol. 3 (1954) pp. 57-121. HARISH-ChANDRA

1. Plancherel formula for the real $2 \times 2$ unimodular group, Proc. Nat. Acad. Sci. U.S.A. vol. 38 (1952) pp. 337-342.

F. I. MAUTNER

1. Unitary representations of locally compact groups I, Ann. of Math. vol. 51 (1950) pp. 1-25.

2. Unitary representations of locally compact groups II, Ann. of Math. 52 (1950) pp. 528556.

3. Fourier analysis and symmetric spaces, Proc. Nat. Acad. Sci. U.S.A. vol. 37 (1951) pp. 529-533.

4. Note on the Fourier inversion formula on groups, Trans. Amer. Math. Soc. vol. 78 (1955) pp. 371-384.

5. Induced representations, Amer. J. Math. vol. 74 (1952) pp. 737-758.

R. E. A. C. PALEY and N. Wiener

1. Fourier transform in the complex domain, Amer. Math. Soc. Colloquium Publications, vol. $19,1934$.

A. Powsner

1. On differential equations of the Sturm-Liouville type on a half-axis, Rec. Math. (Mat. Sbornik) N.S. vol. 23 (1948) pp. 3-52.

\section{SCHWARTZ}

1. Etude des sommes d'exponentielles reelles, Paris, Hermann, 1943.

2. Approximation d'une function quelqonque per des sommes d'exponentielles imaginaires, Ann. Fac. Sci. Univ. Toulouse (4) vol. 6, (1942) pp. 111-174.

3. Theorie generale des fonctions moyenne-periodique, Ann. of Math. vol. 48 (1947), pp. 857-929.

4. Theorie des distributions, vols. I and II, Paris, Hermann, 1950-1951.

5. Analyse et synthese harmonique dans les espaces de distributions, Canadian Journal of Mathematics vol. 3 (1951) pp. 503-512.

6. Theorie des noyaux, Proceedings of the International Congress of Mathematicians, 1950, vol. 1, pp. 220-230.

E. C. TITChmarsh

1. The theory of functions, Oxford University Press, 1932.

2. Theory of Fourier integral, Oxford University Press, 1937.

INSTITUTE FOR AdVANCED STUdy, Princeton, N.J. 